\title{
Laplace equation in a domain with a rectilinear crack: higher order derivatives of the energy with respect to the crack length
}

\author{
Gianni Dal Maso, Gianluca Orlando and Rodica Toader
}

\begin{abstract}
We consider the weak solution of the Laplace equation in a planar domain with a straight crack, prescribing a homogeneous Neumann condition on the crack and a nonhomogeneous Dirichlet condition on the rest of the boundary. For every $k$ we express the $k$-th derivative of the energy with respect to the crack length in terms of a finite number of coefficients of the asymptotic expansion of the solution near the crack tip and of a finite number of other parameters, which only depend on the shape of the domain.
\end{abstract}

Mathematics Subject Classification. 35J20, 35C20, 74R10.

Keywords. Cracked domains, Energy release rate, Higher order derivatives, Asymptotic expansion of solutions.

\section{Contents}

1. Introduction

2. Regularity of the solution with respect to crack length 452

3. Expansions near the crack tip 458

4. Computation of the derivatives of the energy 465

5. The main theorem 470

Acknowledgements $\quad 475$

$\begin{array}{ll}\text { References } & 475\end{array}$

\section{Introduction}

The variational approach to the problem of quasistatic crack growth is based on the iterative solution of incremental minimum problems (see, e.g., [2]). In the antiplane case, when the crack is supposed to grow along a prescribed 
straight line, identified with the $x_{1}$-axis, the $i$-th minimum problem takes the form

$$
\begin{aligned}
\min _{u, s}\left\{\frac{1}{2} \int_{\Omega \backslash \Gamma_{s}}|\nabla u|^{2} \mathrm{~d} x+\operatorname{length}\left(\Gamma_{s}\right) \mid u \in H^{1}\left(\Omega \backslash \Gamma_{s}\right), u=g_{i} \text { on } \partial \Omega \backslash \Gamma_{s},\right. \\
\left.\quad s \geq s_{i-1}\right\},
\end{aligned}
$$

where $\Omega$ is a bounded connected open set of the plane containing the origin, $g_{i}$ is a prescribed function defined on the boundary $\partial \Omega$,

$$
\Gamma_{s}:=\left\{\left(x_{1}, 0\right) \in \bar{\Omega} \mid x_{1} \leq s\right\}
$$

and $\left(u_{i-1}, s_{i-1}\right)$ is a solution of the $(i-1)$-th minimum problem.

Let us examine a single problem of the form (1.1), where for simplicity we denote $g_{i}$ by $g$. For given $s$, we consider the bulk energy $\mathcal{E}(s)$, defined by

$$
\mathcal{E}(s):=\frac{1}{2} \int_{\Omega \backslash \Gamma_{s}}\left|\nabla u_{s}\right|^{2} \mathrm{~d} x
$$

where $u_{s}$ is the solution of the minimum problem

$$
\min _{u}\left\{\frac{1}{2} \int_{\Omega \backslash \Gamma_{s}}|\nabla u|^{2} \mathrm{~d} x \mid u \in H^{1}\left(\Omega \backslash \Gamma_{s}\right), u=g \text { on } \partial \Omega \backslash \Gamma_{s}\right\} .
$$

Then problem (1.1) is equivalent to:

$$
\min _{s}\left\{\mathcal{E}(s)+s \mid s \geq s_{i-1}\right\}
$$

and it is therefore useful to compute all derivatives of the function $s \mapsto \mathcal{E}(s)$, in order to determine the minimality conditions. The main result of this paper is an algorithm to compute

$$
\frac{\mathrm{d}^{k} \mathcal{E}}{\mathrm{d} s^{k}}(s)
$$

Similar results in the case of plane elasticity have been obtained in $[1,5]$.

For simplicity, we will prove the formulas for the derivatives of $\mathcal{E}$ at $s=0$. The starting point of our analysis is the fact that, for every $s, u_{s} \in H^{1}\left(\Omega \backslash \Gamma_{s}\right)$ solves the boundary value problem:

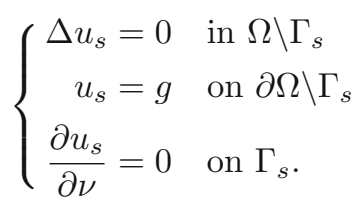

The solution $u_{0}$ to problem (1.3) with $s=0$ will be denoted by $u$. Since $u$ is a harmonic function satisfying a homogeneous Neumann condition on the crack, it has the following asymptotic expansion near the crack tip:

$$
u(\rho, \theta)=\sum_{n=0}^{+\infty}\left[a_{2 n+1} \rho^{\frac{2 n+1}{2}} \sin \left(\frac{2 n+1}{2} \theta\right)+b_{2 n} \rho^{n} \cos (n \theta)\right],
$$

where $(\rho, \theta)$ are polar coordinates, with $\rho>0$ and $-\pi<\theta<\pi$. This is an easy consequence of the standard procedure which transforms a neighborhood of the crack into a half disk using the complex square root (see Proposition 3.1). 
An interesting result, obtained for the first time by Irwin [4], gives an explicit relation between the energy release rate $-\frac{\mathrm{d} \mathcal{E}}{\mathrm{d} s}(0)$ and the coefficient $a_{1}$ of the expansion (1.4) of the solution $u$, called stress intensity factor (see, e.g., $[3])$ :

$$
\frac{\mathrm{d} \mathcal{E}}{\mathrm{d} s}(0)=-\frac{\pi}{4} a_{1}^{2} .
$$

This equality shows that the first derivative of the energy uniquely depends on the local behaviour of the solution near the crack tip and does not depend on the shape of the domain $\Omega$. This is no longer true for higher order derivatives, as noticed by $[1,5]$ in the case of plane elasticity. For $k \geq 2$ we shall see that $\frac{\mathrm{d}^{k} \mathcal{E}}{\mathrm{d} s^{k}}(0)$ depends also on the solutions $v_{\Omega}^{(j)} \in H^{1}\left(\Omega \backslash \Gamma_{0}\right), 1 \leq j \leq k-1$, of the problems

$$
\left\{\begin{aligned}
\Delta v_{\Omega}^{(j)} & =0 & & \text { in } \Omega \backslash \Gamma_{0} \\
v_{\Omega}^{(j)} & =-\rho^{\frac{-2 j+1}{2}} \sin \left(\frac{-2 j+1}{2} \theta\right) & & \text { on } \partial \Omega \backslash \Gamma_{0} \\
\frac{\partial v_{\Omega}^{(j)}}{\partial \nu} & =0 & & \text { on } \Gamma_{0} .
\end{aligned}\right.
$$

These functions have the following asymptotic expansion near the origin:

$$
v_{\Omega}^{(j)}=\sum_{n=0}^{+\infty}\left[c_{2 n+1}^{(j)}(\Omega) \rho^{\frac{2 n+1}{2}} \sin \left(\frac{2 n+1}{2} \theta\right)+d_{2 n}^{(j)}(\Omega) \rho^{n} \cos (n \theta)\right] .
$$

For every $k \geq 1$, let

$$
\lambda_{k}(\Omega)=\left(c_{2 n+1}^{(j)}(\Omega)\right)_{n+j \leq k-1},
$$

considered as an element of $\mathbb{R}^{\frac{k(k-1)}{2}}$.

We are now in a position to state the main result of the paper. Let $\mathcal{A}$ be the collection of the admissible open sets $\Omega$, whose precise definition will be given at the beginning of Sect. 2 .

Theorem 1.1. For every $k \geq 1$ there exists a function

$$
\Psi_{k}: \mathbb{R}^{k} \times \mathbb{R}^{\frac{k(k-1)}{2}} \rightarrow \mathbb{R}
$$

such that for every admissible set $\Omega \in \mathcal{A}$ and for every boundary condition $g \in H^{1}\left(\Omega \backslash \Gamma_{0}\right)$ we have

$$
\frac{\mathrm{d}^{k} \mathcal{E}}{\mathrm{d} s^{k}}(0)=\Psi_{k}\left(\alpha_{k}, \lambda_{k}(\Omega)\right),
$$

where $\lambda_{k}(\Omega)$ are defined in $(1.7)$ and

$$
\alpha_{k}=\left(a_{1}, a_{3}, \ldots, a_{2 k-1}\right),
$$

$a_{i}$ being the coefficients of the asymptotic expansion (1.4). Moreover, for all $\lambda \in \mathbb{R}^{\frac{k(k-1)}{2}}$, the function $\alpha \mapsto \Psi_{k}(\alpha, \lambda)$ is a homogeneous polynomial of degree 2 , and for every $\alpha \in \mathbb{R}^{k}$ the function $\lambda \mapsto \Psi_{k}(\alpha, \lambda)$ is a polynomial of degree $k-1$. 


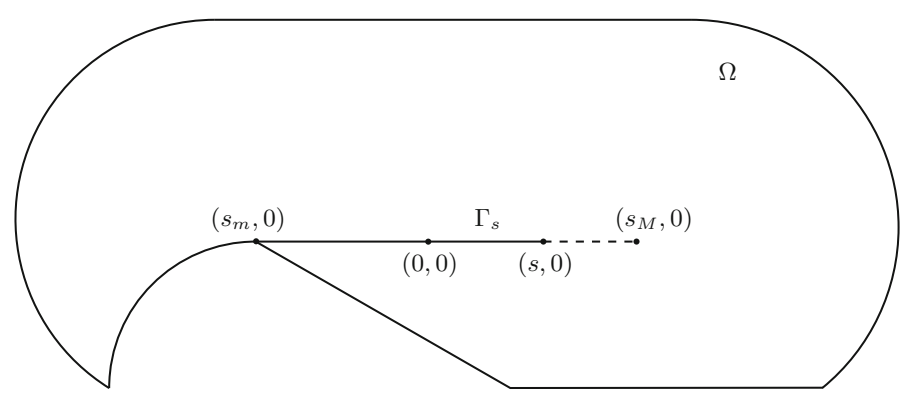

Figure 1. Example of $\Omega \in \mathcal{A}$ with $\Omega^{-}$not Lipschitz

The proof provides also an iterative algorithm for computing $\Psi_{k}$. An essential ingredient is a careful analysis of the harmonic functions $u^{(j)}$ on $\Omega \backslash \Gamma_{0}$, defined as the derivatives of the solution with respect to the crack length:

$$
u^{\prime}:=\lim _{s \rightarrow 0} \frac{u_{s}-u_{0}}{s}, \quad u^{(j)}:=\lim _{s \rightarrow 0} \frac{u_{s}^{(j-1)}-u_{0}^{(j-1)}}{s}, \quad \text { for } j \geq 2 .
$$

A crucial step is the proof of the formulas

$$
u^{(j)}=\sum_{m=1}^{j} a_{-2 m+1}^{(j)}\left[\rho^{\frac{-2 m+1}{2}} \sin \left(\frac{-2 m+1}{2} \theta\right)+v_{\Omega}^{(m)}\right],
$$

which connect the functions $u^{(j)}$ to the shape functions $v_{\Omega}^{(j)}$ defined in (1.5).

\section{Regularity of the solution with respect to crack length}

Let $\Omega$ be a bounded connected open subset of $\mathbb{R}^{2}$ with Lipschitz boundary. Suppose that $\Omega$ contains the origin $0 \in \mathbb{R}^{2}$. To describe the crack lying on the straight line $\mathbb{R} \times\{0\}$, we fix $s_{m}<0<s_{M}$ such that $\left(s_{m}, 0\right) \in \partial \Omega,\left(s_{M}, 0\right) \in \Omega$, and the open segment between $\left(s_{m}, 0\right)$ and $\left(s_{M}, 0\right)$ is contained in $\Omega$. For every $s \in\left(s_{m}, s_{M}\right)$ we set

$$
\Gamma_{s}:=\left\{\left(x_{1}, 0\right) \mid s_{m} \leq x_{1} \leq s\right\} .
$$

Moreover, we suppose that the two sets $\left.\Omega^{ \pm}=\left\{\left(x_{1}, x_{2}\right) \in \Omega\right) \mid \pm x_{2}>0\right\}$ have Lipschitz boundary (Fig. 1). This condition can be slightly generalized by assuming only that the open set $\Omega \backslash \Gamma_{s_{M}}$ can be written as the union of two domains with Lipschitz boundary. The class of Lipschitz domains containing the origin and satisfying the decomposition property mentioned above will be denoted by $\mathcal{A}$. It is easy to see that for every $\Omega \in \mathcal{A}$ and for every $s \in$ $\left(s_{m}, s_{M}\right)$, also the open set $\Omega \backslash \Gamma_{s}$ can be written as the union of two domains with Lipschitz boundary and whose common boundary contains $\Gamma_{s}$. Therefore we can define a trace operator from $H^{1}\left(\Omega \backslash \Gamma_{s}\right)$ into $L^{2}\left(\partial \Omega \backslash \Gamma_{s}\right)$ and employ the Poincaré inequality in $\Omega \backslash \Gamma_{s}$, by considering separately these Lipschitz subdomains (Fig. 1). 
We now fix a function $g \in H^{1}\left(\Omega \backslash \Gamma_{0}\right)$. In order to make precise the notion of solution of problem (1.3), we introduce the space of test functions:

$$
H_{s}:=\left\{\psi \in H^{1}\left(\Omega \backslash \Gamma_{s}\right) \mid \psi=0 \text { on } \partial \Omega \backslash \Gamma_{s}\right\} .
$$

For every $s \in\left(s_{m}, s_{M}\right)$, we say that $u_{s}$ is a solution of (1.3) if $u_{s} \in H^{1}\left(\Omega \backslash \Gamma_{s}\right)$, $u_{s}=g$ on $\partial \Omega \backslash \Gamma_{s}$ and

$$
\int_{\Omega \backslash \Gamma_{s}} \nabla u_{s} \cdot \nabla \psi \mathrm{d} x=0 \quad \text { for every } \psi \in H_{s} .
$$

The solution of problem (1.3) corresponding to $s=0$ will be simply denoted by $u$.

Here we focus our attention on the dependence of the solution $u_{s}$ on the crack length and we study its regularity with respect to the parameter $s$. To do this we reformulate (2.2) as an equation over a fixed domain, by using suitable $s$-dependent diffeomorphisms. To this aim, we fix $\eta \in \mathcal{C}_{c}^{\infty}(\Omega)$ such that $\eta \equiv 1$ in a neighbourhood of the crack tip 0 and consider the map $F_{s}: \mathbb{R}^{2} \rightarrow \mathbb{R}^{2}$ defined by

$$
F_{s}\left(x_{1}, x_{2}\right)=\left(x_{1}+s \eta\left(x_{1}, x_{2}\right), x_{2}\right) .
$$

It is easy to see that there exists $\delta_{0}>0$ such that for every $s \in\left(-\delta_{0}, \delta_{0}\right)$ the map $F_{s}$ is a diffeomorphism and it satisfies the following properties: $F_{s}\left(\Gamma_{0}\right)=$ $\Gamma_{s}$ and $F_{s}$ coincides with the identity near $\partial \Omega$. We may assume that $s_{m}<-\delta_{0}$ and $\delta_{0}<s_{M}$. In addition, we suppose that $\eta$ is radial and satisfies $\eta(\rho)=1$ for $\rho \leq R_{0} / 2$ and $\eta(\rho)=0$ for $\rho \geq R_{0}$, for some $0<R_{0}<\operatorname{dist}(0, \partial \Omega)$.

Let $U_{s}$ be the solution $u_{s}$ of problem (1.3) in the new coordinates, i.e.,

$$
U_{s}:=u_{s} \circ F_{s} \in H^{1}\left(\Omega \backslash \Gamma_{0}\right) .
$$

Remark 2.1. Since $F_{s}$ does not modify the boundary of $\Omega$, surely $U_{s}-g \in H_{0}$, where $H_{0}$ is the space defined in (2.1) for $s=0$. Moreover, by applying the change of coordinates in (2.2), it is straightforward to check that $U_{s}$ solves

$$
\int_{\Omega \backslash \Gamma_{0}}\left(\nabla U_{s}\right)^{T} C_{s} \nabla \xi \mathrm{d} x=0 \quad \text { for all } \xi \in H_{0},
$$

where the matrix $C_{s}$ is defined by

$$
C_{s}(x):=\mathrm{D} F_{s}^{-1}\left(F_{s}(x)\right)\left(\mathrm{D} F_{s}^{-1}\left(F_{s}(x)\right)\right)^{T} \operatorname{det} \mathrm{D} F_{s}(x),
$$

which is a $2 \times 2$ symmetric matrix, smooth with respect to the variables $(s, x)$ and satisfies the uniform ellipticity condition, i.e.,

$$
\zeta^{T} C_{s} \zeta \geq \lambda|\zeta|^{2}
$$

for some $\lambda>0$, for all $x \in \Omega \backslash \Gamma_{0}$, for all $s \in\left(-\delta_{0}, \delta_{0}\right)$ and for all $\zeta \in \mathbb{R}^{2}$. An explicit expression of the matrix $C_{s}$ will be useful. From the definition (2.6) we can compute

$$
C_{s}(x)=\frac{1}{1+s \mathrm{D}_{1} \eta(x)}\left(\begin{array}{cc}
1+s^{2}\left(\mathrm{D}_{2} \eta(x)\right)^{2} & -s \mathrm{D}_{2} \eta(x)\left(1+s \mathrm{D}_{1} \eta(x)\right) \\
-s \mathrm{D}_{2} \eta(x)\left(1+s \mathrm{D}_{1} \eta(x)\right) & \left(1+s \mathrm{D}_{1} \eta(x)\right)^{2}
\end{array}\right) .
$$


Let $C_{s}^{(j)}$ be the $j$-th derivative of this matrix with respect to the parameter $s$; as usual we set $C_{s}^{(0)}=C_{s}$.

We now investigate the regularity of $U_{s}(x)$ with respect to $s$ and $x$. This will be used to deduce the corresponding regularity properties of $u_{s}(x)$.

Theorem 2.2. The function $s \in\left(-\delta_{0}, \delta_{0}\right) \mapsto U_{s} \in H^{1}\left(\Omega \backslash \Gamma_{0}\right)$ is of class $\mathcal{C}^{\infty}$.

Proof. The theorem is a consequence of the Implicit Function Theorem on Banach spaces. In fact, let $H_{0}^{\prime}$ be the dual space of $H_{0}$, and for $s \in\left(-\delta_{0}, \delta_{0}\right)$, let $A_{s}: H_{0} \rightarrow H_{0}^{\prime}$ be the operator defined by

$$
\left\langle A_{s} V, \xi\right\rangle:=\int_{\Omega \backslash \Gamma_{0}} \nabla V^{T} C_{s} \nabla \xi \mathrm{d} x \quad \text { for every } V, \xi \in H_{0} .
$$

For every $s \in\left(-\delta_{0}, \delta_{0}\right)$, the function $V_{s}:=U_{s}-g \in H_{0}$ is the unique solution of the problem

$$
L\left(s, V_{s}\right)=0 \quad \text { in } H_{0}^{\prime},
$$

where the map $L:\left(-\delta_{0}, \delta_{0}\right) \times H_{0} \rightarrow H_{0}^{\prime}$ is defined by

$$
L(s, V):=A_{s} V+A_{s} g .
$$

It is possible to deduce from the smoothness of the matrix $C_{s}$ that the map $L$ is smooth. Moreover, for every $s_{0} \in\left(-\delta_{0}, \delta_{0}\right)$, its derivative with respect to $V$ computed at $\left(s_{0}, 0\right)$ is given by

$$
\frac{\partial L}{\partial V}\left(s_{0}, 0\right)=A_{s_{0}} \in \mathcal{L}\left(H_{0}, H_{0}^{\prime}\right)
$$

and the operator $A_{s_{0}}$ is invertible by the Lax-Milgram Theorem. Hence, by the Implicit Function Theorem, there exists $\delta>0$ such that the locus defined by $L(s, V)=0$ is the graph of a smooth function $\left(s_{0}-\delta, s_{0}+\delta\right) \rightarrow H_{0}$.

The next corollary deals with the regularity of the energy.

Corollary 2.3. The function $s \mapsto \mathcal{E}(s)$ introduced in $(1.2)$ is $\mathcal{C}^{\infty}$.

Proof. Using the change of variables (2.3) we obtain that

$$
\mathcal{E}(s):=\frac{1}{2} \int_{\Omega \backslash \Gamma_{0}}\left(\nabla U_{s}\right)^{T} C_{s} \nabla U_{s} \mathrm{~d} x .
$$

The conclusion follows from Theorem 2.2 and from the fact that $C_{s}$ depends smoothly on $s$.

The following theorem shows that all partial derivatives of $U_{s}$ depend smoothly on $s$.

Theorem 2.4. Let $\omega$ be an open set with $\omega \Subset \Omega \backslash \Gamma_{0}$. Then the function $s \in$ $\left(-\delta_{0}, \delta_{0}\right) \mapsto U_{s} \in H^{m}(\omega)$ is of class $\mathcal{C}^{\infty}$ for every integer $m \geq 1$. 
Proof. Consider an open set $\omega^{\prime}$ such that $\omega \Subset \omega^{\prime} \Subset \Omega \backslash \Gamma_{0}$ and let $\zeta$ be a cut-off function compactly supported in $\omega^{\prime}$ and such that $\zeta \equiv 1$ on $\omega$. After some computations done integrating by parts, one can see that the function $D_{j}\left(\zeta U_{s}\right)$ solves a problem of the form

$$
A_{s} D_{j}\left(\zeta U_{s}\right)+G_{s}=0 \quad \text { in } H^{-1}\left(\omega^{\prime}\right)
$$

where $A_{s}: H_{0}^{1}\left(\omega^{\prime}\right) \rightarrow H^{-1}\left(\omega^{\prime}\right)$ is defined by

$$
\left\langle A_{s} V, \psi\right\rangle:=\int_{\omega^{\prime}}(\nabla V)^{T} C_{s} \nabla \psi \mathrm{d} x \quad \text { for all } V, \psi \in H_{0}^{1}\left(\omega^{\prime}\right),
$$

and where $G_{s}$ is a suitable element of $H^{-1}\left(\omega^{\prime}\right)$, depending smoothly on $s$. Thanks to the smoothness of the matrix $C_{s}$ and to the regularity of the function $s \mapsto U_{s} \in H^{1}\left(\Omega \backslash \Gamma_{0}\right)$ obtained in Theorem 2.2, we deduce that the map $L$ : $\left(-\delta_{0}, \delta_{0}\right) \times H_{0}^{1}\left(\omega^{\prime}\right) \rightarrow H^{-1}\left(\omega^{\prime}\right)$ defined by

$$
L(s, V):=A_{s} V+G_{s}
$$

is smooth. As in the proof of Theorem 2.2, it follows from the Implicit Function Theorem that $s \in\left(-\delta_{0}, \delta_{0}\right) \mapsto D_{j}\left(\zeta U_{s}\right) \in H_{0}^{1}\left(\omega^{\prime}\right)$ is $\mathcal{C}^{\infty}$. This shows that the function $s \in\left(-\delta_{0}, \delta_{0}\right) \mapsto U_{s} \in H^{2}(\omega)$ is $\mathcal{C}^{\infty}$. Arguing by induction, one can prove that for every multi-index $\alpha$, the function $s \mapsto \mathrm{D}^{\alpha}\left(\zeta U_{s}\right)$ belongs to $\mathcal{C}^{\infty}\left(\left(-\delta_{0}, \delta_{0}\right) ; H_{0}^{1}\left(\omega^{\prime}\right)\right)$. This shows that for every integer $m \geq 1$ the function $s \mapsto U_{s}$ belongs to $\mathcal{C}^{\infty}\left(\left(-\delta_{0}, \delta_{0}\right) ; H^{m}(\omega)\right)$.

The next corollary easily follows from Theorem 2.4 and from the Sobolev Embedding Theorem. For every bounded open set $\omega \subset \mathbb{R}^{2}$, the space $\mathcal{C}^{k}(\bar{\omega})$ is endowed with the usual topology of uniform convergence of the functions and all their derivatives up to order $k$.

Corollary 2.5. Let $\omega$ be an open set with $\omega \Subset \Omega \backslash \Gamma_{0}$. Then the function $s \in$ $\left(-\delta_{0}, \delta_{0}\right) \mapsto U_{s} \in C^{k}(\bar{\omega})$ is of class $\mathcal{C}^{\infty}$ for every integer $k \geq 1$.

We can extend the regularity result of Corollary 2.5 also for some open sets $\omega \subset \Omega \backslash \Gamma_{0}$ whose boundary touches $\Gamma_{0}$.

Theorem 2.6. Let $\omega$ be an open subset of $\Omega \backslash \Gamma_{0}$ of the form

$$
\omega:=B_{r}\left(x_{0}\right)^{ \pm}=\left\{\left(x_{1}, x_{2}\right) \in B_{r}\left(x_{0}\right) \mid \pm x_{2}>0\right\},
$$

where $x_{0} \in \Gamma_{0}$. Assume that $0 \notin \bar{\omega}$ and $\omega \Subset \Omega$. Then the function $s \in$ $\left(-\delta_{0}, \delta_{0}\right) \mapsto U_{s} \in \mathcal{C}^{k}(\bar{\omega})$ is of class $\mathcal{C}^{k}$.

Proof. We give the proof only for $B_{r}\left(x_{0}\right)^{+}$. Under our assumptions there exists some $r^{\prime}>r$ such that

$$
\omega^{\prime}:=B_{r^{\prime}}\left(x_{0}\right)^{+} \Subset \Omega \quad \text { and } \quad 0 \notin \overline{\omega^{\prime}} .
$$

Consider the function $\widetilde{U}_{s} \in H^{1}\left(B_{r^{\prime}}\left(x_{0}\right)\right)$ defined by

$$
\widetilde{U}_{s}\left(x_{1}, x_{2}\right):= \begin{cases}U_{s}\left(x_{1}, x_{2}\right) & \text { if } x_{2} \geq 0 \\ U_{s}\left(x_{1},-x_{2}\right) & \text { if } x_{2} \leq 0 .\end{cases}
$$


Let $c_{i j}$ be the coefficients of the matrix $C_{s}$. Since $\eta$ is radial, from (2.7) we see that $c_{11}$ and $c_{22}$ are even in $x_{2}$, while $c_{12}=c_{21}$ is odd in $x_{2}$. Therefore, from (2.5) it follows that $\widetilde{U}_{s}$ solves the problem

$$
\int_{B_{r^{\prime}}\left(x_{0}\right)}\left(\nabla \widetilde{U}_{s}\right)^{T} C_{s} \nabla \psi \mathrm{d} x=0 \quad \text { for all } \psi \in H_{0}^{1}\left(B_{r^{\prime}}\left(x_{0}\right)\right) .
$$

We conclude now as in the proof of Theorem 2.4 and Corollary 2.5.

In view of Theorem 2.2, we are allowed to define the derivative of $U_{s}$ with respect to $s$ by simply taking the limit

$$
U_{s}^{\prime}:=\lim _{h \rightarrow 0} \frac{U_{s+h}-U_{s}}{h}
$$

in the strong topology of $H^{1}\left(\Omega \backslash \Gamma_{0}\right)$. Moreover, for $j \geq 2$, we can define further derivatives of $U_{s}$ by the recursive formula

$$
U_{s}^{(j)}:=\lim _{h \rightarrow 0} \frac{U_{s+h}^{(j-1)}-U_{s}^{(j-1)}}{h},
$$

where the limit is taken in the strong topology of $H^{1}\left(\Omega \backslash \Gamma_{0}\right)$. Observe that, for $j \geq 1$, the function $U_{s}^{(j)}$ is the limit of functions in $H_{0}$, and hence it belongs to $H_{0}$. The convergence in (2.10) and (2.11) takes place in $\mathcal{C}^{k}(\bar{\omega})$ for every open set $\omega \Subset \Omega \backslash \Gamma_{0}$ and also for every half-disk $\omega$ as in Theorem 2.6. As usual we set $U_{s}^{(0)}=U_{s}$ and we adopt the notation $U^{(j)}$ for $U_{s}^{(j)}$ computed at $s=0$.

We can now deal with the regularity of the solution $u_{s}$ with respect to the parameter $s$.

Theorem 2.7. Let $s_{0} \in\left(-\delta_{0}, \delta_{0}\right)$ and let $\omega$ be an open set with $\omega \Subset \Omega$. Assume that either $\omega \Subset \Omega \backslash \Gamma_{s_{0}}$ or that $\omega=B_{r}\left(x_{0}\right)^{ \pm}=\left\{\left(x_{1}, x_{2}\right) \in B_{r}\left(x_{0}\right) \mid \pm x_{2}>0\right\}$ with $x_{0} \in \Gamma_{s_{0}}$ and $\left(s_{0}, 0\right) \notin \bar{\omega}$. Then for every integer $k \geq 0$ there exists $\delta>0$ such that the function $s \in\left(s_{0}-\delta, s_{0}+\delta\right) \mapsto u_{s} \in \mathcal{C}^{k}(\bar{\omega})$ is of class $\mathcal{C}^{k}$.

Proof. The results follow from Corollary 2.5 and Theorem 2.6, by noticing that $u_{s}$ is the composition of $U_{s}$ with the change of coordinates $F_{s}^{-1}$.

The previous results allow us to define the derivatives of $u_{s}$ with respect to the parameter $s$. Define

$$
u_{s}^{\prime}:=\lim _{h \rightarrow 0} \frac{u_{s+h}-u_{s}}{h}
$$

and for every $j \geq 2$, by the recursive formula,

$$
u_{s}^{(j)}:=\lim _{h \rightarrow 0} \frac{u_{s+h}^{(j-1)}-u_{s}^{(j-1)}}{h} .
$$

The convergence in (2.12) and (2.13) takes place in $\mathcal{C}^{k}(\bar{\omega})$ for every $\omega$ as in Theorem 2.7. As usual we set $u_{s}^{(0)}=u_{s}$ and we adopt the notation $u^{(j)}$ for $u_{s}^{(j)}$ computed at $s=0$. 
Proposition 2.8. For every $j \geq 1$, the function $u^{(j)}$ is harmonic on $\Omega \backslash \Gamma_{0}$ and satisfies the Neumann condition $\frac{\partial u^{(j)}}{\partial \nu}=0$ on $\Gamma_{0} \backslash\{0\}$, in the sense that

$$
\lim _{\substack{x \rightarrow x_{0} \\ x \in \Omega \backslash \Gamma_{0}}} \frac{\partial u^{(j)}}{\partial \nu}(x)=0 \quad \text { for every } x_{0} \in \Gamma_{0} \backslash\{0\} .
$$

Proof. The function $u^{\prime}$ is harmonic, because by definition (2.12) it is the uniform limit on compact sets of harmonic functions. It also satisfies the Neumann condition $\frac{\partial u^{\prime}}{\partial \nu}=0$ on $\Gamma_{0} \backslash\{0\}$, since $u_{h}-u$ satisfies the Neumann condition on $\Gamma_{0}$ and the limit in (2.12) takes place in $\mathcal{C}^{k}(\bar{\omega})$ for the half balls $\omega$ considered in Theorem 2.7. By induction, it follows from the same reasons that $u^{(j)}$ is harmonic and satisfies the Neumann condition $\frac{\partial u^{(j)}}{\partial \nu}=0$ on $\Gamma_{0} \backslash\{0\}$.

The following lemma shows the relationship between the derivatives of $U_{s}$ with respect to $s$ and the derivatives of $u_{s}$ with respect to $s$ and $x_{1}$.

Lemma 2.9. For every $j \geq 0$ it holds

$$
U_{s}^{(j)}(x)=\sum_{p=0}^{j}\left(\begin{array}{l}
j \\
p
\end{array}\right) \mathrm{D}_{1}^{p} u_{s}^{(j-p)}\left(F_{s}(x)\right) \eta^{p}(x),
$$

where $\eta$ is the cut-off function involved in the definition (2.3) of the change of coordinates $F_{s}$ and $\mathrm{D}_{1}^{p}$ denotes the derivative of order $p$ in the direction $x_{1}$.

Proof. The simple proof can be done by induction and it is omitted.

Remark 2.10. Since we chose the cut-off function $\eta$ in such a way that it vanishes outside the ball $B_{R_{0}}$, from formula (2.15) we see that $U^{(j)}$ and $u^{(j)}$ coincide out of $B_{R_{0}}$. In particular $u^{(j)}$ is $H^{1}$ far from the crack tip and its trace on $\partial \Omega \backslash \Gamma_{0}$ vanishes.

Moreover, since $u^{(j)}$ is smooth in $\Omega \backslash \Gamma_{0}$ and can be smoothly extended to both sides of $\Gamma_{0} \backslash\{0\}$, we conclude that $u^{(j)}$ belongs to $H^{1}\left(\left(B_{R} \backslash \bar{B}_{\varepsilon}\right) \backslash \Gamma_{0}\right)$, for every $0<\varepsilon<R<\operatorname{dist}(0, \partial \Omega)$.

The following lemma shows that the functions $U_{s}^{(j)}$ are weak solutions of suitable differential equations.

Lemma 2.11. For all $j \geq 0$ we have

$$
\sum_{p=0}^{j}\left(\begin{array}{l}
j \\
p
\end{array}\right) \int_{\Omega \backslash \Gamma_{0}}\left(\nabla U_{s}^{(j-p)}\right)^{T} C_{s}^{(p)} \nabla \xi \mathrm{d} x=0
$$

for all $\xi \in H_{0}$.

Proof. The proof proceeds by induction on $j$. For $j=0$, Eq. (2.16) is exactly Eq. (2.5), which holds true.

Let $j \geq 1$ and assume that the statement is true for $j-1$. Let us prove that it is true for $j$. Equation (2.16) for $j-1$ reads

$$
\sum_{p=0}^{j-1}\left(\begin{array}{c}
j-1 \\
p
\end{array}\right) \int_{\Omega \backslash \Gamma_{0}}\left(\nabla U_{s}^{(j-1-p)}\right)^{T} C_{s}^{(p)} \nabla \xi \mathrm{d} x=0 \quad \text { for all } \xi \in H_{0} .
$$


Thanks to Theorem 2.2, we can derive this equation with respect to $s$. We obtain

$$
\begin{aligned}
0= & \sum_{p=0}^{j-1}\left(\begin{array}{c}
j-1 \\
p
\end{array}\right) \int_{\Omega \backslash \Gamma_{0}}\left[\left(\nabla U_{s}^{(j-p)}\right)^{T} C_{s}^{(p)} \nabla \xi+\left(\nabla U_{s}^{(j-1-p)}\right)^{T} C_{s}^{(p+1)} \nabla \xi\right] \mathrm{d} x \\
= & \int_{\Omega \backslash \Gamma_{0}}\left(\nabla U_{s}^{(j)}\right)^{T} C_{s} \nabla \xi \mathrm{d} x+\sum_{p=1}^{j-1}\left(\begin{array}{c}
j-1 \\
p
\end{array}\right) \int_{\Omega \backslash \Gamma_{0}}\left(\nabla U_{s}^{(j-p)}\right)^{T} C_{s}^{(p)} \nabla \xi \mathrm{d} x \\
& +\sum_{p=0}^{j-2}\left(\begin{array}{c}
j-1 \\
p
\end{array}\right) \int_{\Omega \backslash \Gamma_{0}}\left(\nabla U_{s}^{(j-1-p)}\right)^{T} C_{s}^{(p+1)} \nabla \xi \mathrm{d} x+\int_{\Omega \backslash \Gamma_{0}}\left(\nabla U_{s}\right)^{T} C_{s}^{(j)} \nabla \xi \mathrm{d} x \\
= & \sum_{p=0}^{j}\left(\begin{array}{c}
j \\
p
\end{array}\right) \int_{\Omega \backslash \Gamma_{0}}\left(\nabla U_{s}^{(j-p)}\right)^{T} C_{s}^{(p)} \nabla \xi \mathrm{d} x,
\end{aligned}
$$

which is what we wanted to prove.

\section{Expansions near the crack tip}

In this section we find the asymptotic expansions for the harmonic functions $u$ and $u^{(j)}$ near the crack tip, which coincides with the origin. We start by recalling the result for $u$, which can be obtained by elementary methods of complex analysis.

Proposition 3.1. Let $0<R<\operatorname{dist}(0, \partial \Omega)$. Then (1.4) holds in the cracked ball $B_{R} \backslash \Gamma_{0}$ centred at 0 , and the series in (1.4) converges uniformly on every cracked ball $B_{r} \backslash \Gamma_{0}$, with $0<r<R$.

This expansion is a particular case of a more general result concerning the $u^{(j)}$ proved in Proposition 3.2 (see Remark 3.3). The new difficulty about the $u^{(j)}$ is that, in general, they do not belong to $H^{1}\left(\Omega \backslash \Gamma_{0}\right)$, since they exhibit a stronger singularity at the origin. We remark that Proposition 3.2 is only the starting point in the study of the expansion of $u^{(j)}$, which will be improved in Proposition 3.8 and Theorem 3.9.

Proposition 3.2. Let $j \geq 0$ and $0<R<\operatorname{dist}(0, \partial \Omega)$. Then

$$
u^{(j)}(\rho, \theta)=\sum_{n=-\infty}^{+\infty}\left[a_{2 n+1}^{(j)} \rho^{\frac{2 n+1}{2}} \sin \left(\frac{2 n+1}{2} \theta\right)+b_{2 n}^{(j)} \rho^{n} \cos (n \theta)\right]+c^{(j)} \log \rho
$$

in the cracked ball $B_{R} \backslash \Gamma_{0}$ centred at 0 , and the series in (3.1) converges uniformly on every set of the form $\left(B_{r} \backslash B_{r^{\prime}}\right) \backslash \Gamma_{0}$, with $0<r^{\prime}<r<R$.

Proof. We open the crack by using the bi-holomorphic change of coordinates

$$
\begin{aligned}
\Phi: B_{R} \backslash \Gamma_{0} & \rightarrow B_{\sqrt{R}}^{+}:=\left\{\left(x_{1}, x_{2}\right) \in B_{\sqrt{R}} \mid x_{1}>0\right\} \\
z & \mapsto \sqrt{z},
\end{aligned}
$$


where we identify $\left(x_{1}, x_{2}\right)$ with the complex number $z=x_{1}+i x_{2}$. Notice that the change of coordinates $\Phi$ transforms the part of the crack $\Gamma_{0} \cap \bar{B}_{R}$ into the segment

$$
S:=\left\{\left(x_{1}, x_{2}\right) \in \mathbb{R}^{2} \mid x_{1}=0,-\sqrt{R} \leq x_{2} \leq \sqrt{R}\right\} .
$$

Using the fact that $\Phi$ is bi-holomorphic, it is easy to show that for every $\varepsilon>0$ the function $v:=u^{(j)} \circ \Phi^{-1}$ belongs to $H^{1}\left(\left(B_{\sqrt{R}}^{+} \backslash \bar{B}_{\varepsilon}\right) \backslash \Gamma_{0}\right)$ and by Proposition 2.8 it solves the problem

$$
\begin{cases}\Delta v=0 & \text { in }\left(B_{\sqrt{R}}^{+} \backslash \bar{B}_{\varepsilon}\right) \backslash \Gamma_{0} \\ \frac{\partial v}{\partial \nu}=0 & \text { on } S \backslash B_{\varepsilon}\end{cases}
$$

By reflecting the function $v$, we can define a harmonic function on the whole annulus $B_{\sqrt{R}} \backslash \bar{B}_{\varepsilon}$ :

$$
w\left(x_{1}, x_{2}\right)= \begin{cases}v\left(x_{1}, x_{2}\right) & \text { if } x_{1} \geq 0 \\ v\left(-x_{1}, x_{2}\right) & \text { if } x_{1}<0\end{cases}
$$

This construction can be repeated for every $\varepsilon>0$, hence we can extend the function $w$ to a harmonic function on the punctured disk $B_{\sqrt{R}} \backslash\{0\}$. Therefore there exists a constant $c^{(j)}$ such that the function $w(x)-c^{(j)} \log |x|$ is the imaginary part of a holomorphic function $f$ on the punctured disk $B_{\sqrt{R}} \backslash\{0\}$. The function $f$ can be expanded in a Laurent series

$$
f(z)=\sum_{n=-\infty}^{+\infty}\left(a_{n}^{(j)}+i b_{n}^{(j)}\right) z^{n}, \quad a_{n}^{(j)}, b_{n}^{(j)} \in \mathbb{R},
$$

which uniformly converges on every annulus centred at 0 and strictly contained in the punctured disk $B_{\sqrt{R}} \backslash\{0\}$. By taking the imaginary part in (3.2), we obtain an expansion for the function $w$ and hence for the function $v$ in polar coordinates in the half disk $B_{\sqrt{R}}^{+}$

$$
v(\rho, \theta)=\sum_{n=-\infty}^{+\infty}\left[a_{n}^{(j)} \rho^{n} \sin (n \theta)+b_{n}^{(j)} \rho^{n} \cos (n \theta)\right]+c^{(j)} \log \rho .
$$

By applying the holomorphic change of coordinates $\Phi$ to this expansion, we get exactly the expansion (3.1) in the statement of the proposition, since the coefficients $a_{n}^{(j)}$ for $n$ even and $b_{n}^{(j)}$ for $n$ odd must vanish because the Neumann condition $\left.\frac{\partial u^{(j)}}{\partial \theta}\right|_{\theta= \pm \pi}=0$ is satisfied.

Remark 3.3. In the case $j=0$, the function $u$ belongs to $H^{1}\left(B_{R} \backslash \Gamma_{0}\right)$, hence $v \in H^{1}\left(B_{\sqrt{R}}^{+}\right)$. This implies that $w \in H^{1}\left(B_{\sqrt{R}}\right)$. As a consequence $c^{(0)}=0$ and the function $f$ is holomorphic in the whole disk $B_{\sqrt{R}}$, and therefore (3.2) reduces to a Taylor expansion, i.e. $a_{n}^{(0)}=0, b_{n}^{(0)}=0$ for $n<0$. This leads to the classical expansion (1.4) for $u$. 
For future use, we state the following corollary to Proposition 3.2, regarding the regularity properties of a function whose expansion of type (3.1) starts from a positive index.

Corollary 3.4. Let us fix $j \geq 0, k \geq 0$, let $a_{2 n+1}^{(j)}, b_{2 n}^{(j)}$ be as in Proposition 3.2, and let

$$
v_{k}(\rho, \theta):=\sum_{n=k}^{+\infty}\left[a_{2 n+1}^{(j)} \rho^{\frac{2 n+1}{2}} \sin \left(\frac{2 n+1}{2} \theta\right)+b_{2 n}^{(j)} \rho^{n} \cos (n \theta)\right] .
$$

Then $v_{k} \in H^{k+1}\left(B_{r} \backslash \Gamma_{0}\right)$ for every $0<r<R$.

The proof is based on the following two technical lemmas.

Lemma 3.5. Let $D$ be a domain in $\mathbb{C}$, and let $f: D \rightarrow \mathbb{C}$ be a holomorphic function. Let $u=\operatorname{Re} f$ and $v=\operatorname{Im} f$. Assume that $f, f^{\prime}, \ldots, f^{(k)} \in L^{2}(D)$. Then $u, v \in H^{k}(D)$.

Proof. The thesis follows from the fact that for every $h \geq 1$ and for every $0 \leq$ $\alpha_{1}, \alpha_{2}, \beta_{1}, \beta_{2} \leq h$ with $\left|\alpha_{1}-\beta_{1}\right|=1,\left|\alpha_{2}-\beta_{2}\right|=1$, and $\alpha_{1}+\alpha_{2}=\beta_{1}+\beta_{2}=\bar{h}$, we have

$$
f^{(h)}=\sigma\left[\mathrm{D}_{1}^{\alpha_{1}} \mathrm{D}_{2}^{\alpha_{2}} v+i \mathrm{D}_{1}^{\beta_{1}} \mathrm{D}_{2}^{\beta_{2}} v\right]
$$

for a suitable constant $\sigma \in\{ \pm 1, \pm i\}$. This can be easily proved by induction on $h$, using the fact that for every holomorphic function $g$ we have $g^{\prime}=\mathrm{D}_{1} g=$ $-i \mathrm{D}_{2} g$.

Lemma 3.6. Let $f: B_{\sqrt{R}} \rightarrow \mathbb{C}$ be a holomorphic function and let $\varphi: B_{R} \backslash \Gamma_{0} \rightarrow$ $\mathbb{C}$ be defined by $\varphi(z):=f(\sqrt{z})$. Assume that $f(z)=z^{2 k} g(z)$ for some $k \geq 0$ and some holomorphic function $g: B_{\sqrt{R}} \rightarrow \mathbb{C}$. Then $\varphi, \varphi^{\prime}, \ldots, \varphi^{(k+1)} \in L^{2}\left(B_{r} \backslash \Gamma_{0}\right)$ for every $r<R$.

Proof. We observe that $\varphi(z)=z^{k} \psi(z)$, where $\psi(z)=g(\sqrt{z})$. We can easily prove by induction that for every $h \geq 1$ we have

$$
\psi^{(h)}(z)=\sum_{m=1}^{h} c_{m}^{h} g^{(m)}(\sqrt{z}) z^{\frac{1}{2} m-h},
$$

for suitable constants $c_{m}^{h}$. Let us fix $0<r<R$. Since $g$ is holomorphic in $B_{\sqrt{R}}$, the functions $g^{(m)}$ are bounded in $B_{\sqrt{r}}$. Hence there exists a constant $C_{h}$ such that

$$
\left|\psi^{(h)}(z)\right| \leq C_{h}|z|^{\frac{1}{2}-h} \quad \text { for every } z \in B_{r} \text {, for every } 1 \leq h \leq k+1 .
$$

Since $\varphi(z)=z^{k} \psi(z)$, for every $1 \leq h \leq k$, by Leibniz's rule we have the estimate

$$
\begin{aligned}
\left|\varphi^{(h)}(z)\right| & \leq \sum_{m=1}^{h} C_{m, h, k}|z|^{k-h+m}|z|^{\frac{1}{2}-m}+C_{0, h, k}|z|^{k-h} \\
& \leq C|z|^{k-h} \quad \text { for every } z \in B_{r},
\end{aligned}
$$


which shows that $\varphi^{(h)}$ is bounded on $B_{r}$ for $1 \leq h \leq k$. As for $h=k+1$, by Leibniz's rule we have

$$
\left|\varphi^{(k+1)}(z)\right| \leq \sum_{m=1}^{k+1} C_{m, k}|z|^{-1+m}|z|^{\frac{1}{2}-m} \leq C|z|^{-\frac{1}{2}} \quad \text { for every } z \in B_{r},
$$

which shows that $\varphi^{(k+1)} \in L^{2}\left(B_{r}\right)$.

Proof of Corollary 3.4 Since the function $f$ introduced in the proof of Proposition 3.2 has the expansion (3.2), we have that the function

$$
f_{2 k}(z)=\sum_{n=2 k}^{+\infty}\left(a_{n}^{(j)}+i b_{n}^{(j)}\right) z^{n}
$$

is holomorphic in $B_{\sqrt{R}}$. As the coefficients $a_{n}^{(j)}$ for $n$ even and $b_{n}^{(j)}$ for $n$ odd vanish (see again the proof of Proposition 3.2), the function $v_{k}$ is the imaginary part of $f_{2 k}(\sqrt{z})$. The conclusion follows now from Lemmas 3.5 and 3.6.

The next step is to show that indeed the series in (3.1) has finitely many nonzero terms for $n$ negative. To do this, we prove an estimate on $u^{(j)}$ which shows that it belongs to the dual of a suitable subspace of $H^{j-1}\left(B_{R} \backslash \Gamma_{0}\right)$.

Let $B_{R}^{ \pm}:=\left\{\left(x_{1}, x_{2}\right) \in B_{R} \mid \pm x_{2}>0\right\}$ and let $\mathcal{T}$ be the class of test functions $\varphi \in \mathcal{C}^{\infty}\left(B_{R} \backslash \Gamma_{0}\right) \cap \mathcal{C}^{\infty}\left(\bar{B}_{R}^{+}\right) \cap \mathcal{C}^{\infty}\left(\bar{B}_{R}^{-}\right)$, which vanish on a neighbourhood of $\partial B_{R} \backslash \Gamma_{0}$ and on a neighbourhood of the crack tip 0 . For every $\varphi \in \mathcal{T}$ and for every $k \geq 0$ we set

$$
\left\|\mathrm{D}^{k} \varphi\right\|_{L^{2}\left(B_{R} \backslash \Gamma_{0}\right)}:=\left(\sum_{|\alpha|=k}\left\|\mathrm{D}^{\alpha} \varphi\right\|_{L^{2}\left(B_{R} \backslash \Gamma_{0}\right)}^{2}\right)^{\frac{1}{2}} .
$$

Lemma 3.7. Let $R>0$ with $\eta=1$ on $B_{R}$. For every $j \geq 1$ there exists a positive constant $C_{j}>0$ such that the estimate

$$
\left|\int_{B_{R} \backslash \Gamma_{0}} u^{(j)} \varphi \mathrm{d} x\right| \leq C_{j}\left\|\mathrm{D}^{j-1} \varphi\right\|_{L^{2}\left(B_{R} \backslash \Gamma_{0}\right)}
$$

holds for every $\varphi \in \mathcal{T}$.

Proof. Let us prove the claim by induction on $j$. For the case $j=1$, simply observe that by Lemma 2.9 we have that

$$
u^{\prime}=U^{\prime}-\mathrm{D}_{1} u \in L^{2}\left(B_{R} \backslash \Gamma_{0}\right)
$$

and therefore estimate (3.4) holds.

Let $j \geq 2$ and suppose that the claim is true for $j-p$ with $1 \leq p \leq j-1$. From Lemma 2.9 we deduce that

$$
u^{(j)}=U^{(j)}-\sum_{p=1}^{j}\left(\begin{array}{l}
j \\
p
\end{array}\right) \mathrm{D}_{1}^{p} u^{(j-p)} \quad \text { in } B_{R} \backslash \Gamma_{0} .
$$


Fix a test function $\varphi \in \mathcal{T}$. Since $U^{(j)} \in L^{2}\left(B_{R} \backslash \Gamma_{0}\right)$, by applying Hölder inequality and Poincaré inequality to all the derivatives of $\varphi$ of order less or equal than $j-2$, we get the estimate

$$
\begin{aligned}
\left|\int_{B_{R} \backslash \Gamma_{0}} U^{(j)} \varphi \mathrm{d} x\right| & \leq\left\|U^{(j)}\right\|_{L^{2}\left(B_{R} \backslash \Gamma_{0}\right)}\|\varphi\|_{L^{2}\left(B_{R} \backslash \Gamma_{0}\right)} \\
& \leq\left\|U^{(j)}\right\|_{L^{2}\left(B_{R} \backslash \Gamma_{0}\right)}\left\|\mathrm{D}^{j-1} \varphi\right\|_{L^{2}\left(B_{R} \backslash \Gamma_{0}\right)} .
\end{aligned}
$$

Let us estimate the other terms of the sum, integrating by parts with respect to the variable $x_{1}$ and using the induction hypothesis:

$$
\begin{aligned}
\left|\int_{B_{R} \backslash \Gamma_{0}}\left(\mathrm{D}_{1}^{p} u^{(j-p)}\right) \varphi \mathrm{d} x\right| & =\left|\int_{B_{R} \backslash \Gamma_{0}} u^{(j-p)} \mathrm{D}_{1}^{p} \varphi \mathrm{d} x\right| \leq C_{j-p}\left\|\mathrm{D}^{j-p-1} \mathrm{D}_{1}^{p} \varphi\right\|_{L^{2}\left(B_{R} \backslash \Gamma_{0}\right)} \\
& \leq C_{j-p}\left\|\mathrm{D}^{j-1} \varphi\right\|_{L^{2}\left(B_{R} \backslash \Gamma_{0}\right)} .
\end{aligned}
$$

This concludes the proof.

We now improve the result obtained in Proposition 3.2. Indeed we show that in the asymptotic expansion (3.1) all coefficients of $u^{(j)}$ with $n<-j$ vanish.

Proposition 3.8. Let $0<R<\operatorname{dist}(0, \partial \Omega)$. Then

$$
u^{(j)}(\rho, \theta)=\sum_{n=-j}^{+\infty}\left[a_{2 n+1}^{(j)} \rho^{\frac{2 n+1}{2}} \sin \left(\frac{2 n+1}{2} \theta\right)+b_{2 n}^{(j)} \rho^{n} \cos (n \theta)\right]+c^{(j)} \log \rho
$$

in the cracked ball $B_{R} \backslash \Gamma_{0}$, and the series in (3.5) converges uniformly on every set of the form $\left(B_{r} \backslash B_{r^{\prime}}\right) \backslash \Gamma_{0}$, with $0<r^{\prime}<r<R$.

Proof. From Proposition 3.2 we know that (3.1) holds. Let us prove that for every $n \geq j$ we have $a_{-(2 n+1)}^{(j)}=0$. In order to do this, fix $0<r^{\prime}<R^{\prime}<R$ such that $\eta=1$ on $B_{R^{\prime}}$ and let $\varphi \in \mathcal{T}$ be a test function of the form

$$
\varphi(\rho, \theta)=\psi(\rho) \sin \left(\frac{2 n+1}{2} \theta\right)
$$

where $\psi$ is a nonzero smooth function, with $\operatorname{supp} \psi \Subset\left(r^{\prime}, R^{\prime}\right)$ and $\psi a_{-(2 n+1)}^{(j)} \leq$ 0 . Define the rescaled function

$$
\varphi_{\varepsilon}(\rho, \theta):=\varphi\left(\frac{\rho}{\varepsilon}, \theta\right)=\psi\left(\frac{\rho}{\varepsilon}\right) \sin \left(\frac{2 n+1}{2} \theta\right) .
$$

Estimate (3.4) holds for $\varphi_{\varepsilon}$, and therefore

$$
C_{j}\left\|\mathrm{D}^{j-1} \varphi_{\varepsilon}\right\|_{L^{2}\left(B_{R^{\prime}} \backslash \Gamma_{0}\right)} \geq \int_{\varepsilon r^{\prime}}^{\varepsilon R^{\prime}} \int_{-\pi}^{\pi} u^{(j)}(\rho, \theta) \psi\left(\frac{\rho}{\varepsilon}\right) \sin \left(\frac{2 n+1}{2} \theta\right) \rho \mathrm{d} \theta \mathrm{d} \rho .
$$

By the uniform convergence of the series in (3.1) and by the orthogonality of the trigonometric functions, from the previous inequality we obtain 


$$
\begin{aligned}
C_{j}\left\|\mathrm{D}^{j-1} \varphi_{\varepsilon}\right\|_{L^{2}\left(B_{R^{\prime}} \backslash \Gamma_{0}\right)} \geq & \pi \int_{\varepsilon r^{\prime}}^{\varepsilon R^{\prime}} \psi\left(\frac{\rho}{\varepsilon}\right)\left[-a_{-(2 n+1)}^{(j)} \rho^{-\frac{2 n+1}{2}}+a_{2 n+1}^{(j)} \rho^{\frac{2 n+1}{2}}\right] \rho \mathrm{d} \rho \\
= & \pi \varepsilon^{2} \int_{r^{\prime}}^{R^{\prime}} \psi(\rho)\left[-a_{-(2 n+1)}^{(j)} \rho^{-\frac{2 n+1}{2}} \varepsilon^{-\frac{2 n+1}{2}}\right] \rho \mathrm{d} \rho \\
& +\pi \varepsilon^{2} \int_{r^{\prime}}^{R^{\prime}} \psi(\rho)\left[a_{2 n+1}^{(j)} \rho^{\frac{2 n+1}{2}} \varepsilon^{\frac{2 n+1}{2}}\right] \rho \mathrm{d} \rho .
\end{aligned}
$$

On the other hand

$$
\left\|\mathrm{D}^{j-1} \varphi_{\varepsilon}\right\|_{L^{2}\left(B_{R} \backslash \Gamma_{0}\right)}=\varepsilon^{2-j}\left\|\mathrm{D}^{j-1} \varphi\right\|_{L^{2}\left(B_{R} \backslash \Gamma_{0}\right)}
$$

and hence we have that

$$
\begin{aligned}
C_{j}\left\|\mathrm{D}^{j-1} \varphi\right\|_{L^{2}\left(B_{R^{\prime}} \backslash \Gamma_{0}\right) \geq} & \pi \varepsilon^{2-\frac{2 n+1}{2}-(2-j)} \int_{r^{\prime}}^{R^{\prime}} \psi(\rho)\left[-a_{-(2 n+1)}^{(j)} \rho^{-\frac{2 n+1}{2}}\right] \rho \mathrm{d} \rho \\
& +\pi \varepsilon^{2+\frac{2 n+1}{2}-(2-j)} \int_{r^{\prime}}^{R^{\prime}} \psi(\rho)\left[a_{2 n+1}^{(j)} \rho^{\frac{2 n+1}{2}}\right] \rho \mathrm{d} \rho .
\end{aligned}
$$

If $a_{-(2 n+1)}^{(j)}$ were different from zero, the right-hand side in (3.6) would diverge to $+\infty$ for $\varepsilon \rightarrow 0^{+}$. Therefore $a_{-(2 n+1)}=0$.

Arguing in the same way and using a test function of the form

$$
\varphi(\rho, \theta)=\psi(\rho) \cos (n \theta),
$$

we obtain that $b_{-2 n}^{(j)}=0$ for $n>j$.

We are now in a position to prove the final result on the asymptotic expansion of $u^{(j)}$. The improvement with respect to Proposition 3.8 is that the coefficients of the cosines with negative index vanish as well as the coefficient of the logarithmic term.

Theorem 3.9. Let $0<R<\operatorname{dist}(0, \partial \Omega)$. Then

$$
u^{(j)}(\rho, \theta)=\sum_{n=-j}^{+\infty} a_{2 n+1}^{(j)} \rho^{\frac{2 n+1}{2}} \sin \left(\frac{2 n+1}{2} \theta\right)+\sum_{n=0}^{+\infty} b_{2 n}^{(j)} \rho^{n} \cos (n \theta)
$$

in the cracked ball $B_{R} \backslash \Gamma_{0}$, and the series in (3.7) converges uniformly on every set of the form $\left(B_{r} \backslash B_{r^{\prime}}\right) \backslash \Gamma_{0}$, with $0<r^{\prime}<r<R$.

Proof. Besides the binomial coefficient, we shall use the Pochhammer symbol defined by

$$
(x)_{p}:=x(x-1) \cdots(x-p+1)
$$

for every $x \in \mathbb{R}$ and every integer $p \geq 1$. We set also $(x)_{0}=1$.

Fix $0<r<R^{\prime}<R$ where $R^{\prime}$ is such that $\eta=1$ on $B_{R^{\prime}}$. Using Proposition 3.8 and Corollary 3.4, it is straightforward to check that for every 
$1 \leq p \leq j$ the function $\mathrm{D}_{1}^{p} u^{(j-p)}$ has the following expansion in $B_{r} \backslash \Gamma_{0}$ :

$$
\begin{aligned}
\mathrm{D}_{1}^{p} u^{(j-p)}= & \sum_{n=-j+p}^{p-1}\left(\frac{2 n+1}{2}\right)_{p} a_{2 n+1}^{(j-p)} \rho^{\frac{2 n+1}{2}-p} \sin \left(\left(\frac{2 n+1}{2}-p\right) \theta\right) \\
& +\sum_{n=-j+p}^{-1}(n)_{p} b_{2 n}^{(j-p)} \rho^{n-p} \cos ((n-p) \theta) \\
& -(-1)^{p}(p-1) ! c^{(j-p)} \rho^{-p} \cos (p \theta)+v_{j, p},
\end{aligned}
$$

where $v_{j, p} \in H^{1}\left(B_{r} \backslash \Gamma_{0}\right)$. In the formula above $c^{(0)}=0$ by Remark 3.3. By Lemma 2.9, on $B_{r} \backslash \Gamma_{0}$ we have

$$
\begin{aligned}
U^{(j)}= & \sum_{p=0}^{j}\left(\begin{array}{l}
j \\
p
\end{array}\right) \mathrm{D}_{1}^{p} u^{(j-p)} \\
= & \sum_{p=0}^{j} \sum_{n=-j+p}^{p-1}\left(\begin{array}{l}
j \\
p
\end{array}\right)\left(\frac{2 n+1}{2}\right)_{p} a_{2 n+1}^{(j-p)} \rho^{\frac{2 n+1}{2}-p} \sin \left(\left(\frac{2 n+1}{2}-p\right) \theta\right) \\
& +\sum_{p=0}^{j} \sum_{n=-j+p}^{-1}\left(\begin{array}{l}
j \\
p
\end{array}\right)(n)_{p} b_{2 n}^{(j-p)} \rho^{n-p} \cos ((n-p) \theta) \\
& -\sum_{p=1}^{j-1}(-1)^{p}(p-1) !\left(\begin{array}{l}
j \\
p
\end{array}\right) c^{(j-p)} \rho^{-p} \cos (p \theta) \\
& +c^{(j)} \log \rho+w_{j},
\end{aligned}
$$

where $w_{j} \in H^{1}\left(B_{r} \backslash \Gamma_{0}\right)$. We set $V^{(j)}:=U^{(j)}-w_{j}$. From the previous formula, we obtain that the function

$$
V^{(j)}=\sum_{n=1}^{j} \alpha_{n}^{(j)} \rho^{\frac{-2 n+1}{2}} \sin \left(\frac{-2 n+1}{2} \theta\right)+\sum_{n=1}^{j} \beta_{n}^{(j)} \rho^{-n} \cos (n \theta)+c^{(j)} \log \rho,
$$

where

$$
\begin{gathered}
\alpha_{n}^{(j)}:=\sum_{p=0}^{j}\left(\begin{array}{l}
j \\
p
\end{array}\right)\left(\frac{2(p-n)+1}{2}\right)_{p} a_{2(p-n)+1}^{(j-p)}, \\
\beta_{n}^{(j)}:=\sum_{p=0}^{n-1}\left(\begin{array}{l}
j \\
p
\end{array}\right)(p-n)_{p} b_{2(p-n)}^{(j-p)}-(-1)^{n}(n-1) !\left(\begin{array}{c}
j \\
n
\end{array}\right) c^{(j-n)} .
\end{gathered}
$$

Since $U^{(j)} \in H^{1}\left(\Omega \backslash \Gamma_{0}\right)$ by Theorem 2.2, we conclude that $V^{(j)} \in H^{1}\left(B_{r} \backslash \Gamma_{0}\right)$.

Let us prove that $\beta_{j}^{(j)}=0$. To this aim we consider $\mathrm{D}_{\rho} V^{(j)}$, which can be written as

$$
\mathrm{D}_{\rho} V^{(j)}=-j \beta_{j}^{(j)} \rho^{-j-1}(\cos (j \theta)+h(\rho, \theta))
$$

where $h(\rho, \theta) \rightarrow 0$ as $\rho \rightarrow 0$, uniformly with respect to $\theta$. Since $\mathrm{D}_{\rho} V^{(j)} \in$ $L^{2}\left(B_{r} \backslash \Gamma_{0}\right)$, it follows that $\beta_{j}^{(j)}=0$. Then we can prove in a similar way that 
$\alpha_{j}^{(j)}=0, \beta_{j-1}^{(j)}=0, \ldots, \alpha_{1}^{(j)}=0$, and $c^{(j)}=0$. Therefore, by (3.10), for all $1 \leq n \leq j$ we have

$$
\sum_{p=0}^{n-1}\left(\begin{array}{l}
j \\
p
\end{array}\right)(p-n)_{p} b_{2(p-n)}^{(j-p)}=0
$$

From (3.12) it is easy to see by induction on $j$ that $b_{-2 n}^{(j)}=0$ for all $1 \leq n \leq j$. The conclusion follows from (3.5).

Remark 3.10. As a byproduct of the proof of Theorem 3.9, we obtain that

$$
\sum_{p=0}^{j}\left(\begin{array}{l}
j \\
p
\end{array}\right)\left(\frac{2(p-n)+1}{2}\right)_{p} a_{2(p-n)+1}^{(j-p)}=0 \quad \text { for all } 1 \leq n \leq j,
$$

as a consequence of (3.9) and from the equality $\alpha_{n}^{(j)}=0$ for $1 \leq n \leq j$. These relations will be useful in the proof of the main theorem.

\section{Computation of the derivatives of the energy}

In this section we express the derivatives $\frac{\mathrm{d}^{k} \mathcal{E}}{\mathrm{d} s^{k}}(0)$ of the energy $\mathcal{E}(s)$ defined in (1.2) in terms of the coefficients of the asymptotic expansion (1.4) of the solution $u$ for $s=0$ and in terms of the coefficients of the corresponding expansions (3.7) of the $j$-th derivatives $u^{(j)}$ of $u_{s}$ with respect to $s$. We already know that $\mathcal{E}$ is $\mathcal{C}^{\infty}$ by Corollary 2.3 .

Proposition 4.1. For every $k \geq 1$ we have

$$
\frac{\mathrm{d}^{k} \mathcal{E}}{\mathrm{d} s^{k}}(s)=\frac{1}{2} \sum_{p=1}^{k}\left(\begin{array}{l}
k \\
p
\end{array}\right) \int_{\Omega \backslash \Gamma_{0}}\left(\nabla U_{s}^{(k-p)}\right)^{T} C_{s}^{(p)} \nabla U_{s} \mathrm{~d} x
$$

for every $s \in\left(-\delta_{0}, \delta_{0}\right)$.

Proof. In order to prove formula (4.1), we proceed by induction on $k$. For the case $k=1$, simply differentiate formula (2.8) with respect to $s$ :

$$
\frac{\mathrm{d} \mathcal{E}}{\mathrm{d} s}(s)=\int_{\Omega \backslash \Gamma_{s}}\left(\nabla U_{s}^{\prime}\right)^{T} C_{s} \nabla U_{s} \mathrm{~d} x+\frac{1}{2} \int_{\Omega \backslash \Gamma_{s}}\left(\nabla U_{s}\right)^{T} C_{s}^{\prime} \nabla U_{s} \mathrm{~d} x .
$$

Since $U_{s}^{\prime}$ belongs to the space $H_{0}$ introduced in $(2.1)$, we can use it as a test function for problem (2.5). Since $C_{s}$ is symmetric, this implies that

$$
\frac{\mathrm{d} \mathcal{E}}{\mathrm{d} s}(s)=\frac{1}{2} \int_{\Omega \backslash \Gamma_{s}}\left(\nabla U_{s}\right)^{T} C_{s}^{\prime} \nabla U_{s} \mathrm{~d} x,
$$

which is formula (4.1) for $k=1$.

Let us suppose by induction that the statement is true for $k-1$, and let us prove that it is true for $k$. By differentiating with respect to $s$ the following formula

$$
\frac{\mathrm{d}^{k-1} \mathcal{E}}{\mathrm{d} s^{k-1}}(s)=\frac{1}{2} \sum_{p=1}^{k-1}\left(\begin{array}{c}
k-1 \\
p
\end{array}\right) \int_{\Omega \backslash \Gamma_{0}}\left(\nabla U_{s}^{(k-1-p)}\right)^{T} C_{s}^{(p)} \nabla U_{s} \mathrm{~d} x
$$


we obtain

$$
\begin{aligned}
\frac{\mathrm{d}^{k} \mathcal{E}}{\mathrm{d} s^{k}}(s)=\frac{1}{2} \sum_{p=1}^{k-1}\left(\begin{array}{c}
k-1 \\
p
\end{array}\right) \int_{\Omega \backslash \Gamma_{0}} & {\left[\left(\nabla U_{s}^{(k-p)}\right)^{T} C_{s}^{(p)} \nabla U_{s}+\left(\nabla U_{s}^{(k-1-p)}\right)^{T} C_{s}^{(p+1)} \nabla U_{s}\right.} \\
& \left.+\left(\nabla U_{s}^{(k-1-p)}\right)^{T} C_{s}^{(p)} \nabla U_{s}^{\prime}\right] \mathrm{d} x .
\end{aligned}
$$

We now use $U_{s}^{\prime}$ as a test function for problem (2.16) solved by $U_{s}^{(k-1)}$ and then $U_{s}^{(k-1)}$ as a test function for the problem solved by $U_{s}^{\prime}$, and we obtain that

$$
\begin{aligned}
\frac{1}{2} \sum_{p=1}^{k-1}\left(\begin{array}{c}
k-1 \\
p
\end{array}\right) \int_{\Omega \backslash \Gamma_{0}}\left(\nabla U_{s}^{(k-1-p)}\right)^{T} C_{s}^{(p)} \nabla U_{s}^{\prime} \mathrm{d} x & =-\frac{1}{2} \int_{\Omega \backslash \Gamma_{0}}\left(\nabla U_{s}^{(k-1)}\right)^{T} C_{s} \nabla U_{s}^{\prime} \mathrm{d} x \\
& =\frac{1}{2} \int_{\Omega \backslash \Gamma_{0}}\left(\nabla U_{s}^{(k-1)}\right)^{T} C_{s}^{\prime} \nabla U_{s} \mathrm{~d} x
\end{aligned}
$$

which, substituted in the expression of $\frac{\mathrm{d}^{k} \mathcal{E}}{\mathrm{d} s^{k}}$, yields

$$
\begin{aligned}
& \frac{\mathrm{d}^{k} \mathcal{E}}{\mathrm{d} s^{k}}(s)=\frac{1}{2} \sum_{p=1}^{k-1}\left(\begin{array}{c}
k-1 \\
p
\end{array}\right) \int_{\Omega \backslash \Gamma_{0}}\left[\left(\nabla U_{s}^{(k-p)}\right)^{T} C_{s}^{(p)} \nabla U_{s}\right. \\
& \left.+\left(\nabla U_{s}^{(k-1-p)}\right)^{T} C_{s}^{(p+1)} \nabla U_{s}\right] \mathrm{d} x \\
& +\frac{1}{2} \int_{\Omega \backslash \Gamma_{0}}\left(\nabla U_{s}^{(k-1)}\right)^{T} C_{s}^{\prime} \nabla U_{s} \mathrm{~d} x \\
& =\frac{k-1}{2} \int_{\Omega \backslash \Gamma_{0}}\left(\nabla U_{s}^{(k-1)}\right)^{T} C_{s}^{\prime} \nabla U_{s} \mathrm{~d} x \\
& +\frac{1}{2} \sum_{p=2}^{k-1}\left(\begin{array}{c}
k-1 \\
p
\end{array}\right) \int_{\Omega \backslash \Gamma_{0}}\left(\nabla U_{s}^{(k-p)}\right)^{T} C_{s}^{(p)} \nabla U_{s} \mathrm{~d} x \\
& +\frac{1}{2} \sum_{p=2}^{k-1}\left(\begin{array}{l}
k-1 \\
p-1
\end{array}\right) \int_{\Omega \backslash \Gamma_{0}}\left(\nabla U_{s}^{(k-p)}\right)^{T} C_{s}^{(p)} \nabla U_{s} \mathrm{~d} x \\
& +\frac{1}{2} \int_{\Omega \backslash \Gamma_{0}}\left(\nabla U_{s}\right)^{T} C_{s}^{(k)} \nabla U_{s} \mathrm{~d} x \\
& +\frac{1}{2} \int_{\Omega \backslash \Gamma_{0}}\left(\nabla U_{s}^{(k-1)}\right)^{T} C_{s}^{\prime} \nabla U_{s} \mathrm{~d} x \\
& =\frac{1}{2} \sum_{p=1}^{k}\left(\begin{array}{l}
k \\
p
\end{array}\right) \int_{\Omega \backslash \Gamma_{0}}\left(\nabla U_{s}^{(k-p)}\right)^{T} C_{s}^{(p)} \nabla U_{s} \mathrm{~d} x .
\end{aligned}
$$

This concludes the proof.

In order to expand formula (4.1), we compute the derivatives of the matrix $C_{s}$ with respect to $s$. 
Lemma 4.2. The following equalities hold

$$
C_{0}^{\prime}=\left(\begin{array}{cc}
-\mathrm{D}_{1} \eta & -\mathrm{D}_{2} \eta \\
-D_{2} \eta & \mathrm{D}_{1} \eta
\end{array}\right), \quad C_{0}^{(k)}=\left(\begin{array}{cc}
(-1)^{k} k !\left(\mathrm{D}_{1} \eta\right)^{k-2}|\nabla \eta|^{2} & 0 \\
0 & 0
\end{array}\right)
$$

for every $k \geq 2$.

Proof. From the expression of $C_{s}$ written in (2.7), we have that

$$
C_{s}=\mathrm{D} F_{s}^{-1}\left(\mathrm{D} F_{s}^{-1}\right)^{T} \operatorname{det} \mathrm{D} F_{s}=\frac{1}{1+s \mathrm{D}_{1} \eta} M_{s}
$$

where

$$
M_{s}=\left(\begin{array}{cc}
1+s^{2} \mathrm{D}_{2} \eta & -s \mathrm{D}_{2} \eta\left(1+s \mathrm{D}_{1} \eta\right) \\
-s \mathrm{D}_{2} \eta\left(1+s \mathrm{D}_{1} \eta\right) & \left(1+s \mathrm{D}_{1} \eta\right)^{2}
\end{array}\right) .
$$

Hence, by differentiating with respect to $s$ we obtain that

$$
C_{s}^{\prime}=\frac{-\mathrm{D}_{1} \eta}{\left(1+s \mathrm{D}_{1} \eta\right)^{2}} M_{s}+\frac{1}{1+s \mathrm{D}_{1} \eta} M_{s}^{\prime}
$$

which computed in $s=0$ gives the formula we wanted to prove for the first derivative.

Instead of proving the statement of the lemma, it is convenient to show by induction a stronger result, i.e., that for every $k \geq 2$

$C_{s}^{(k)}=\frac{(-1)^{k} k !\left(\mathrm{D}_{1} \eta\right)^{k}}{\left(1+s \mathrm{D}_{1} \eta\right)^{k+1}} M_{s}+\frac{(-1)^{k-1} k !\left(\mathrm{D}_{1} \eta\right)^{k-1}}{\left(1+s \mathrm{D}_{1} \eta\right)^{k}} M_{s}^{\prime}+\frac{(-1)^{k} k !\left(\mathrm{D}_{1} \eta\right)^{k-2}}{2\left(1+s \mathrm{D}_{1} \eta\right)^{k-1}} M_{s}^{\prime \prime}$.

The base case $k=2$ is obtained by simply differentiating formula (4.2) with respect to $s$. The inductive step follows easily from the fact that the matrix $M_{s}^{\prime \prime}$ does not actually depend on $s$, since the entries of $M_{s}$ are polynomials of degree 2 with respect to $s$.

Proposition 4.3. For every $k \geq 1$, we have

$$
\begin{aligned}
\frac{\mathrm{d}^{k} \mathcal{E}}{\mathrm{d} s^{k}}(0)= & \frac{k}{2} \sum_{j=0}^{k-1}\left(\begin{array}{c}
k-1 \\
j
\end{array}\right) \int_{0}^{R_{0}} \eta^{\prime}(\rho) \eta(\rho)^{j} \int_{-\pi}^{\pi}\left[-\mathrm{D}_{1}^{j+1} u^{(k-j-1)} \mathrm{D}_{\rho} u\right. \\
& \left.+\frac{1}{\rho} \mathrm{D}_{2} \mathrm{D}_{1}^{j} u^{(k-j-1)} \mathrm{D}_{\theta} u\right] \rho \mathrm{d} \theta \mathrm{d} \rho
\end{aligned}
$$

where $R_{0}$ is the radius of the ball which supports the cutoff function $\eta$.

Proof. Let us start by computing $\frac{\mathrm{d}^{k} \mathcal{E}}{\mathrm{d} s^{k}}$ at 0 using formula (4.1) and employing the expression of the derivatives of $C_{s}$ found in Lemma 4.2:

$$
\begin{aligned}
\frac{\mathrm{d}^{k} \mathcal{E}}{\mathrm{d} s^{k}}(0)= & \frac{1}{2} \sum_{p=1}^{k}\left(\begin{array}{l}
k \\
p
\end{array}\right) \int_{\Omega \backslash \Gamma_{0}}\left(\nabla U^{(k-p)}\right)^{T} C_{0}^{(p)} \nabla u \mathrm{~d} x \\
= & \frac{k}{2} \int_{\Omega \backslash \Gamma_{0}}\left[-\mathrm{D}_{1} \eta \mathrm{D}_{1} U^{(k-1)} \mathrm{D}_{1} u-\mathrm{D}_{2} \eta \mathrm{D}_{1} U^{(k-1)} \mathrm{D}_{2} u\right. \\
& \left.-\mathrm{D}_{2} \eta \mathrm{D}_{2} U^{(k-1)} \mathrm{D}_{1} u+\mathrm{D}_{1} \eta \mathrm{D}_{2} U^{(k-1)} \mathrm{D}_{2} u\right] \mathrm{d} x \\
& +\frac{1}{2} \sum_{p=2}^{k}\left(\begin{array}{l}
k \\
p
\end{array}\right) \int_{\Omega \backslash \Gamma_{0}}(-1)^{p} p !\left(\mathrm{D}_{1} \eta\right)^{p-2}|\nabla \eta|^{2} \mathrm{D}_{1} U^{(k-p)} \mathrm{D}_{1} u \mathrm{~d} x .
\end{aligned}
$$


By using Lemma 2.9 and expanding the derivatives, we get

$$
\begin{aligned}
\frac{\mathrm{d}^{k} \mathcal{E}}{\mathrm{d} s^{k}}(0)= & \frac{k}{2} \sum_{j=0}^{k-1}\left(\begin{array}{c}
k-1 \\
j
\end{array}\right) \int_{\Omega \backslash \Gamma_{0}}\left[-\mathrm{D}_{1} \eta \mathrm{D}_{1} u \mathrm{D}_{1}^{j+1} u^{(k-1-j)} \eta^{j}\right. \\
& -j\left(\mathrm{D}_{1} \eta\right)^{2} \mathrm{D}_{1} u \mathrm{D}_{1}^{j} u^{(k-1-j)} \eta^{j-1} \\
& -\mathrm{D}_{2} \eta \mathrm{D}_{2} u \mathrm{D}_{1}^{j+1} u^{(k-1-j)} \eta^{j} \\
& -j \mathrm{D}_{1} \eta \mathrm{D}_{2} \eta \mathrm{D}_{2} u \mathrm{D}_{1}^{j} u^{(k-1-j)} \eta^{j-1} \\
& -\mathrm{D}_{2} \eta \mathrm{D}_{1} u \mathrm{D}_{2} \mathrm{D}_{1}^{j} u^{(k-1-j)} \eta^{j} \\
& -j\left(\mathrm{D}_{2} \eta\right)^{2} \mathrm{D}_{1} u \mathrm{D}_{1}^{j} u^{(k-1-j)} \eta^{j-1} \\
& +\mathrm{D}_{1} \eta \mathrm{D}_{2} u \mathrm{D}_{2} \mathrm{D}_{1}^{j} u^{(k-1-j)} \eta^{j} \\
& \left.+j \mathrm{D}_{1} \eta \mathrm{D}_{2} \eta \mathrm{D}_{2} u \mathrm{D}_{1}^{j} u^{(k-1-j)} \eta^{j-1}\right] \mathrm{d} x \\
& +\frac{1}{2} \sum_{p=2}^{k} \sum_{j=0}^{k-p}\left(\begin{array}{l}
k \\
p
\end{array}\right)\left(\begin{array}{c}
k-p \\
j
\end{array}\right)(-1)^{p} p ! \int_{\Omega \backslash \Gamma_{0}}\left[\left(\mathrm{D}_{1} \eta\right)^{p-2}|\nabla \eta|^{2} \mathrm{D}_{1} u \mathrm{D}_{1}^{j+1} u^{(k-p-j)} \eta^{j}\right. \\
& \left.+j\left(\mathrm{D}_{1} \eta\right)^{p-1}|\nabla \eta|^{2} \mathrm{D}_{1} u \mathrm{D}_{1}^{j} u^{(k-p-j)} \eta^{j-1}\right] \mathrm{d} x .
\end{aligned}
$$

After some algebraic manipulations, we obtain

$$
\begin{aligned}
\frac{\mathrm{d}^{k} \mathcal{E}}{\mathrm{d} s^{k}}(0)= & \frac{k}{2} \sum_{j=0}^{k-1}\left(\begin{array}{c}
k-1 \\
j
\end{array}\right) \int_{\Omega \backslash \Gamma_{0}}\left[-\mathrm{D}_{1} \eta \mathrm{D}_{1} u \mathrm{D}_{1}^{j+1} u^{(k-1-j)} \eta^{j}\right. \\
& -\mathrm{D}_{2} \eta \mathrm{D}_{2} u \mathrm{D}_{1}^{j+1} u^{(k-1-j)} \eta^{j} \\
& \left.-\mathrm{D}_{2} \eta \mathrm{D}_{1} u \mathrm{D}_{2} \mathrm{D}_{1}^{j} u^{(k-1-j)} \eta^{j}+\mathrm{D}_{1} \eta \mathrm{D}_{2} u \mathrm{D}_{2} \mathrm{D}_{1}^{j} u^{(k-1-j)} \eta^{j}\right] \mathrm{d} x \\
& -\frac{k}{2} \sum_{j=1}^{k-1}\left(\begin{array}{c}
k-1 \\
j
\end{array}\right) \int_{\Omega \backslash \Gamma_{0}} j|\nabla \eta|^{2} \mathrm{D}_{1} u \mathrm{D}_{1}^{j} u^{(k-1-j)} \eta^{j-1} \mathrm{~d} x \\
& +\frac{1}{2} \sum_{p=2}^{k} \sum_{j=0}^{k-p}\left(\begin{array}{c}
k \\
p
\end{array}\right)\left(\begin{array}{c}
k-p \\
j
\end{array}\right)(-1)^{p} p ! \int_{\Omega \backslash \Gamma_{0}}\left(\mathrm{D}_{1} \eta\right)^{p-2}|\nabla \eta|^{2} \mathrm{D}_{1} u \mathrm{D}_{1}^{j+1} u^{(k-p-j)} \eta^{j} \mathrm{~d} x \\
& +\frac{1}{2} \sum_{p=2}^{k-1} \sum_{j=1}^{k-p}\left(\begin{array}{c}
k \\
p
\end{array}\right)\left(\begin{array}{c}
k-p \\
j
\end{array}\right)(-1)^{p} p ! \int_{\Omega \backslash \Gamma_{0}} j\left(\mathrm{D}_{1} \eta\right)^{p-1}|\nabla \eta|^{2} \mathrm{D}_{1} u \mathrm{D}_{1}^{j} u^{(k-p-j)} \eta^{j-1} \mathrm{~d} x \\
= & I+I I+I I I+I V .
\end{aligned}
$$

where $I, I I, I I I, I V$ are the four sums appearing in the formula above. Let us consider the term of the sum $I I I$ corresponding to $p=2$ :

$$
\begin{aligned}
& \frac{1}{2} \sum_{j=0}^{k-2}\left(\begin{array}{c}
k \\
2
\end{array}\right)\left(\begin{array}{c}
k-2 \\
j
\end{array}\right) 2 ! \int_{\Omega \backslash \Gamma_{0}}|\nabla \eta|^{2} \mathrm{D}_{1} u \mathrm{D}_{1}^{j+1} u^{(k-2-j)} \eta^{j} \mathrm{~d} x \\
& \quad=\frac{k}{2} \sum_{j=0}^{k-2}\left(\begin{array}{c}
k-1 \\
j+1
\end{array}\right)(j+1) \int_{\Omega \backslash \Gamma_{0}}|\nabla \eta|^{2} \mathrm{D}_{1} u \mathrm{D}_{1}^{j+1} u^{(k-2-j)} \eta^{j} \mathrm{~d} x \\
& \quad=\frac{k}{2} \sum_{j=1}^{k-1}\left(\begin{array}{c}
k-1 \\
j
\end{array}\right) j \int_{\Omega \backslash \Gamma_{0}}|\nabla \eta|^{2} \mathrm{D}_{1} u \mathrm{D}_{1}^{j} u^{(k-1-j)} \eta^{j-1} \mathrm{~d} x=-I I .
\end{aligned}
$$


Let us now consider all the other terms of the sum $I I I$ :

$$
\begin{aligned}
\frac{1}{2} \sum_{p=3}^{k} & \sum_{j=0}^{k-p}\left(\begin{array}{c}
k \\
p
\end{array}\right)\left(\begin{array}{c}
k-p \\
j
\end{array}\right)(-1)^{p} p ! \int_{\Omega \backslash \Gamma_{0}}\left(\mathrm{D}_{1} \eta\right)^{p-2}|\nabla \eta|^{2} \mathrm{D}_{1} u \mathrm{D}_{1}^{j+1} u^{(k-p-j)} \eta^{j} \mathrm{~d} x \\
= & \frac{1}{2} \sum_{p=2}^{k-1} \sum_{j=0}^{k-p-1}\left(\begin{array}{c}
k \\
p+1
\end{array}\right)\left(\begin{array}{c}
k-p-1 \\
j
\end{array}\right)(-1)^{p+1}(p+1) ! \\
& \times \int_{\Omega \backslash \Gamma_{0}}\left(\mathrm{D}_{1} \eta\right)^{p-1}|\nabla \eta|^{2} \mathrm{D}_{1} u \mathrm{D}_{1}^{j+1} u^{(k-p-1-j)} \eta^{j} \mathrm{~d} x \\
= & \frac{1}{2} \sum_{p=2}^{k-1} \sum_{j=1}^{k-p}\left(\begin{array}{c}
k \\
p+1
\end{array}\right)\left(\begin{array}{c}
k-p-1 \\
j-1
\end{array}\right)(-1)^{p+1}(p+1) ! \\
& \times \int_{\Omega \backslash \Gamma_{0}}\left(\mathrm{D}_{1} \eta\right)^{p-1}|\nabla \eta|^{2} \mathrm{D}_{1} u \mathrm{D}_{1}^{j} u^{(k-p-j)} \eta^{j-1} \mathrm{~d} x \\
= & -\frac{1}{2} \sum_{p=2}^{k-1} \sum_{j=1}^{k-p}\left(\begin{array}{c}
k \\
p
\end{array}\right)\left(\begin{array}{c}
k-p \\
j
\end{array}\right)(-1)^{p} p ! j \\
& \times \int_{\Omega \backslash \Gamma_{0}}\left(\mathrm{D}_{1} \eta\right)^{p-1}|\nabla \eta|^{2} \mathrm{D}_{1} u \mathrm{D}_{1}^{j} u^{(k-p-j)} \eta^{j-1} \mathrm{~d} x=-I V .
\end{aligned}
$$

It follows that

$$
\begin{aligned}
\frac{\mathrm{d}^{k} \mathcal{E}}{\mathrm{d} s^{k}}(0)= & \frac{k}{2} \sum_{j=0}^{k-1}\left(\begin{array}{c}
k-1 \\
j
\end{array}\right) \int_{\Omega \backslash \Gamma_{0}}\left[-\mathrm{D}_{1} \eta \mathrm{D}_{1} u \mathrm{D}_{1}^{j+1} u^{(k-1-j)} \eta^{j}\right. \\
& -\mathrm{D}_{2} \eta \mathrm{D}_{2} u \mathrm{D}_{1}^{j+1} u^{(k-1-j)} \eta^{j} \\
& \left.-\mathrm{D}_{2} \eta \mathrm{D}_{1} u \mathrm{D}_{2} \mathrm{D}_{1}^{j} u^{(k-1-j)} \eta^{j}+\mathrm{D}_{1} \eta \mathrm{D}_{2} u \mathrm{D}_{2} \mathrm{D}_{1}^{j} u^{(k-1-j)} \eta^{j}\right] \mathrm{d} x .
\end{aligned}
$$

Now we integrate in polar coordinates to deduce

$$
\begin{aligned}
\frac{\mathrm{d}^{k} \mathcal{E}}{\mathrm{d} s^{k}}(0)= & \frac{k}{2} \sum_{j=0}^{k-1}\left(\begin{array}{c}
k-1 \\
j
\end{array}\right) \int_{0}^{R_{0}} \int_{-\pi}^{\pi} \eta^{\prime}(\rho) \eta(\rho)^{j}\left[-\mathrm{D}_{1} u \cos \theta \mathrm{D}_{1}^{j+1} u^{(k-1-j)}\right. \\
& -\mathrm{D}_{2} u \sin \theta \mathrm{D}_{1}^{j+1} u^{(k-1-j)} \\
& \left.-\mathrm{D}_{1} u \sin \theta \mathrm{D}_{2} \mathrm{D}_{1}^{j} u^{(k-1-j)}+\mathrm{D}_{2} u \cos \theta \mathrm{D}_{2} \mathrm{D}_{1}^{j} u^{(k-1-j)}\right] \rho \mathrm{d} \theta \mathrm{d} \rho \\
= & \frac{k}{2} \sum_{j=0}^{k-1}\left(\begin{array}{c}
k-1 \\
j
\end{array}\right) \int_{0}^{R_{0}} \eta^{\prime}(\rho) \eta(\rho)^{j} \int_{-\pi}^{\pi}\left[-\mathrm{D}_{1}^{j+1} u^{(k-j-1)} \mathrm{D}_{\rho} u\right. \\
& \left.+\frac{1}{\rho} \mathrm{D}_{2} \mathrm{D}_{1}^{j} u^{(k-j-1)} \mathrm{D}_{\theta} u\right] \rho \mathrm{d} \theta \mathrm{d} \rho .
\end{aligned}
$$

This concludes the proof.

The following theorem allows us to express the $k$-th derivative of the energy in terms of the following coefficients of the expansions of the solution $u$ and of its derivatives $u^{(j)}$ with respect to the crack length: 


$$
\begin{array}{ccccc}
a_{-2 k+3}^{(k-1)} & a_{-2 k+5}^{(k-2)} & \cdots & a_{-1}^{(1)} & a_{1} \\
a_{-2 k+5}^{(k-1)} & a_{-2 k+7}^{(k-2)} & \cdots & a_{1}^{(1)} & a_{3} \\
\vdots & \vdots & & \vdots & \vdots \\
a_{-1}^{(k-1)} & a_{1}^{(k-2)} & \cdots & a_{2 k-5}^{(1)} & a_{2 k-3} \\
a_{1}^{(k-1)} & a_{3}^{(k-2)} & \cdots & a_{2 k-3}^{(1)} & a_{2 k-1} .
\end{array}
$$

Theorem 4.4. For every $k \geq 1$ we have

$$
\frac{\mathrm{d}^{k} \mathcal{E}}{\mathrm{d} s^{k}}(0)=\pi \sum_{j=0}^{k-1} \sum_{n=-k+j+1}^{j}\left(\begin{array}{c}
k \\
j+1
\end{array}\right)\left(\frac{2 n+1}{2}\right)_{j+2} a_{2 n+1}^{(k-j-1)} a_{2(j-n)+1} .
$$

Proof. Formula (4.3) easily follows from the expression of $\frac{\mathrm{d}^{k} \mathcal{E}}{\mathrm{d} s^{k}}(0)$ found in Proposition 4.3, using the uniform convergence of the expansions (3.1) of the functions $u^{(j)}$ to justify the integration term by term, and employing simple trigonometric identities to integrate the single terms.

\section{The main theorem}

Formula (4.3) also holds for $k=1$. Hence the first derivative of the energy is given by

$$
\frac{\mathrm{d} \mathcal{E}}{\mathrm{d} s}(0)=-\frac{\pi}{4} a_{1}^{2}
$$

This is a well known result, which shows that the first derivative of the energy uniquely depends on the local behaviour of the solution near the crack tip. We now study the case $k \geq 2$. We shall see that the higher order derivatives of the energy depend not only on the local behaviour of the solution near the crack tip, but also on the shape of $\Omega \backslash \Gamma_{0}$. Indeed, we shall show that these derivatives can be expressed in terms of a finite number of coefficients of the asymptotic expansion of the solution and of a finite number of other parameters, which only depend on the shape of the domain. In order to do this, we need to introduce some technical tools.

Definition 5.1. For every $j \geq 1$ let $v_{\Omega}^{(j)} \in H^{1}\left(\Omega \backslash \Gamma_{0}\right)$ be the weak solution of the problem

$$
\left\{\begin{aligned}
\Delta v_{\Omega}^{(j)} & =0 & & \text { in } \Omega \backslash \Gamma_{0} \\
v_{\Omega}^{(j)} & =-\rho^{\frac{-2 j+1}{2}} \sin \left(\frac{-2 j+1}{2} \theta\right) & & \text { on } \partial \Omega \backslash \Gamma_{0} \\
\frac{\partial v_{\Omega}^{(j)}}{\partial \nu} & =0 & & \text { on } \Gamma_{0} .
\end{aligned}\right.
$$

Remark 5.2. Notice that the function $v_{\Omega}^{(j)}$ depends only on the shape of the domain $\Omega \backslash \Gamma_{0}$, and does not depend on the boundary value $g$ prescribed in problem (1.3). 
In view of Proposition 3.1, the function $v_{\Omega}^{(j)}$ can be expanded in a series near the crack tip:

$$
v_{\Omega}^{(j)}=\sum_{n=0}^{+\infty}\left[c_{2 n+1}^{(j)}(\Omega) \rho^{\frac{2 n+1}{2}} \sin \left(\frac{2 n+1}{2} \theta\right)+d_{2 n}^{(j)}(\Omega) \rho^{n} \cos (n \theta)\right] .
$$

The following proposition provides some equalities which will be used to prove the main theorem, combined with those found in Remark 3.10.

Proposition 5.3. For every $j \geq 1$ and for every $n \geq 0$ we have

$$
a_{2 n+1}^{(j)}=\sum_{m=1}^{j} a_{-2 m+1}^{(j)} c_{2 n+1}^{(m)}(\Omega) .
$$

Proof. Define on $\Omega \backslash \Gamma_{0}$ the function

$$
w^{(j)}:=u^{(j)}-\sum_{m=1}^{j} a_{-2 m+1}^{(j)} \rho^{\frac{-2 m+1}{2}} \sin \left(\frac{-2 m+1}{2} \theta\right)=: u^{(j)}+\sigma^{(j)} .
$$

We now show that $w^{(j)}$ is the variational solution of a suitable boundary value problem. First of all, the function $w^{(j)}$ belongs to $H^{1}\left(\Omega \backslash \Gamma_{0}\right)$. Indeed, the function $u^{(j)}$ is in $H^{1}$ away from the crack tip by Remark 2.10, and the sum $\sigma^{(j)}$ is also smooth away from the crack tip. Moreover, from (3.7), we deduce that $w^{(j)}$ has the following expansion

$$
w^{(j)}=\sum_{n=0}^{+\infty}\left[a_{2 n+1}^{(j)} \rho^{\frac{2 n+1}{2}} \sin \left(\frac{2 n+1}{2} \theta\right)+b_{2 n}^{(j)} \rho^{n} \cos (n \theta)\right],
$$

which belongs to $H^{1}$ near the crack tip, by Corollary 3.4. We conclude that $w^{(j)} \in H^{1}\left(\Omega \backslash \Gamma_{0}\right)$. We observe that $w^{(j)}$ is harmonic in $\Omega \backslash \Gamma_{0}$, since by Proposition 2.8 the function $u^{(j)}$ is harmonic and by direct check the sum $\sigma^{(j)}$ is harmonic too. Let us see which boundary conditions are satisfied by $w^{(j)}$. Both $u^{(j)}$ and the sum $\sigma^{(j)}$ satisfy the Neumann condition on $\Gamma_{0} \backslash\{0\}$, by Proposition 2.8 and by direct check respectively. Let us consider now the trace of $w^{(j)}$ on $\partial \Omega \backslash \Gamma_{0}$. By Remark 2.10 the trace of $u^{(j)}$ vanishes on $\partial \Omega \backslash \Gamma_{0}$, hence the trace of $w^{(j)}$ on $\partial \Omega \backslash \Gamma_{0}$ is $\sigma^{(j)}$. In conclusion, $w^{(j)} \in H^{1}\left(\Omega \backslash \Gamma_{0}\right)$ is a weak solution of the problem

$$
\left\{\begin{aligned}
\Delta w^{(j)} & =0 & & \text { in } \Omega \backslash \Gamma_{0} \\
w^{(j)} & =-\sum_{m=1}^{j} a_{-2 m+1}^{(j)} \rho^{\frac{-2 m+1}{2}} \sin \left(\frac{-2 m+1}{2} \theta\right) & & \text { on } \partial \Omega \backslash \Gamma_{0} \\
\frac{\partial w^{(j)}}{\partial \nu} & =0 & & \text { on } \Gamma_{0} .
\end{aligned}\right.
$$

By the uniqueness of the solution of this problem, we have that

$$
w^{(j)}=\sum_{m=1}^{j} a_{-2 m+1}^{(j)} v_{\Omega}^{(m)}
$$


and hence by (5.3)

$$
u^{(j)}=\sum_{m=1}^{j} a_{-2 m+1}^{(j)}\left[\rho^{\frac{-2 m+1}{2}} \sin \left(\frac{-2 m+1}{2} \theta\right)+v_{\Omega}^{(m)}\right] .
$$

Comparing the expansions of both sides of the last equation, we get the thesis.

For every $k \geq 2$, let

$$
\lambda_{k}(\Omega)=\left(c_{2 n+1}^{(j)}(\Omega)\right)_{n+j \leq k-1},
$$

considered as an element of $\mathbb{R}^{\frac{k(k-1)}{2}}$. Written in a convenient way, the entries of $\lambda_{k}(\Omega)$ make up the following triangular matrix

$$
\begin{array}{cccc}
c_{1}^{(1)}(\Omega) & c_{1}^{(2)}(\Omega) & \cdots & c_{1}^{(k-2)}(\Omega) c_{1}^{(k-1)}(\Omega) \\
c_{3}^{(1)}(\Omega) & c_{3}^{(2)}(\Omega) & \cdots & c_{3}^{(k-2)}(\Omega) \\
\vdots & \vdots & . \cdot & \\
c_{2 k-5}^{(1)}(\Omega) & c_{2 k-5}^{(2)}(\Omega) & & \\
c_{2 k-3}^{(1)}(\Omega) & &
\end{array}
$$

We are now ready to prove the main result of the paper.

Proof of Theorem 1.1 Fix $\Omega \in \mathcal{A}$ and $g \in H^{1}\left(\Omega \backslash \Gamma_{0}\right)$. In view of (5.1) it suffices to prove the theorem for $k \geq 2$. In formula (4.3) the $k$-th derivative of the energy was expressed as a linear combination of the following terms:

$$
\begin{array}{ccccc}
a_{-2 k+3}^{(k-1)} a_{2 k-1} & a_{-2 k+5}^{(k-2)} a_{2 k-1} & \cdots & a_{-1}^{(1)} a_{2 k-1} & a_{1} a_{2 k-1} \\
a_{-2 k+5}^{(k-1)} a_{2 k-3} & a_{-2 k+7}^{(k-2)} a_{2 k-3} & \cdots & a_{1}^{(1)} a_{2 k-3} & a_{3} a_{2 k-3} \\
\vdots & \vdots & & \vdots & \vdots \\
a_{-1}^{(k-1)} a_{3} & a_{1}^{(k-2)} a_{3} & \cdots & a_{2 k-5}^{(1)} a_{3} & a_{2 k-3} a_{3} \\
a_{1}^{(k-1)} a_{1} & a_{3}^{(k-2)} a_{1} & \cdots & a_{2 k-3}^{(1)} a_{1} & a_{2 k-1} a_{1}
\end{array}
$$

We collect the terms different from $a_{1}, \cdots, a_{2 k-1}$ in the matrix

$$
A_{k}=\left(\begin{array}{ccccc}
a_{-2 k+3}^{(k-1)} & a_{-2 k+5}^{(k-2)} & \cdots & a_{-3}^{(2)} & a_{-1}^{(1)} \\
a_{-2 k+5}^{(k-1)} & a_{-2 k+7}^{(k-2)} & \cdots & a_{-1}^{(2)} & a_{1}^{(1)} \\
\vdots & \vdots & & \vdots & \vdots \\
a_{-1}^{(k-1)} & a_{1}^{(k-2)} & \cdots & a_{2 k-7}^{(2)} & a_{2 k-5}^{(1)} \\
a_{1}^{(k-1)} & a_{3}^{(k-2)} & \cdots & a_{2 k-5}^{(2)} & a_{2 k-3}^{(1)}
\end{array}\right) \in \mathbb{R}^{k \times(k-1)}
$$

With this notation, formula (4.3) for the $k$-th derivative of the energy can be written in the following compact way

$$
\frac{\mathrm{d}^{k} \mathcal{E}}{\mathrm{d} s^{k}}(0)=E_{k}\left(A_{k}, \alpha_{k}\right)+F_{k}\left(\alpha_{k}, \alpha_{k}\right),
$$


where $E_{k}: \mathbb{R}^{k \times(k-1)} \times \mathbb{R}^{k} \rightarrow \mathbb{R}$ and $F_{k}: \mathbb{R}^{k} \times \mathbb{R}^{k} \rightarrow \mathbb{R}$ are suitable bilinear maps.

We now prove that we can express the entries of $A_{k}$ in terms of $\alpha_{k-1}:=$ $\left(a_{1}, \cdots, a_{2 k-3}\right)$ and $\lambda_{k}(\Omega)$. Indeed, we show by induction that for every $k \geq 2$ there exists a map

$$
\Lambda_{k}: \mathbb{R}^{k-1} \times \mathbb{R}^{\frac{k(k-1)}{2}} \rightarrow \mathbb{R}^{k \times(k-1)}
$$

such that

$$
A_{k}=\Lambda_{k}\left(\alpha_{k-1}, \lambda_{k}(\Omega)\right)
$$

Moreover we shall see that for every $\lambda \in \mathbb{R}^{\frac{k(k-1)}{2}}$, the function $\alpha \mapsto \Lambda_{k}(\alpha, \lambda)$ is linear, and for every $\alpha \in \mathbb{R}^{k-1}$, the function $\lambda \mapsto \Lambda_{k}(\alpha, \lambda)$ is a polynomial of degree $k-1$. To do this, we will make use of the relations found in Remark 3.10 and in Proposition 5.3

$$
\begin{gathered}
a_{-2 n+1}^{(k-1)}=-\sum_{p=1}^{k-1}\left(\begin{array}{c}
k-1 \\
p
\end{array}\right)\left(\frac{2(p-n)+1}{2}\right)_{p} a_{2(p-n)+1}^{(k-1-p)}, \quad \text { for all } 1 \leq n \leq k-1, \\
a_{2 n+1}^{(k-1)}=\sum_{m=1}^{k-1} a_{-2 m+1}^{(k-1)} c_{2 n+1}^{(m)}(\Omega), \quad \text { for all } n \geq 0 .
\end{gathered}
$$

Base case: Let us define $\Lambda_{2}: \mathbb{R} \times \mathbb{R} \rightarrow \mathbb{R}^{2 \times 1}$. From Eqs. (5.8) and (5.9) with $k=2$, we deduce that

$$
a_{-1}^{(1)}=-\frac{1}{2} a_{1} \quad \text { and } \quad a_{1}^{(1)}=a_{-1}^{(1)} c_{1}^{(1)}(\Omega)=-\frac{1}{2} a_{1} c_{1}^{(1)}(\Omega) .
$$

Hence $A_{2}=\Lambda_{2}\left(a_{1}, c_{1}^{(1)}(\Omega)\right)$, where

$$
\Lambda_{2}(\alpha, \lambda):=\left(\begin{array}{c}
-\frac{1}{2} \alpha \\
-\frac{1}{2} \alpha \lambda
\end{array}\right) .
$$

The map $\Lambda_{2}$ is linear with respect to $\alpha$ and it is a polynomial of degree 1 with respect to $\lambda$.

Inductive step: Suppose that there exists a map

$$
\Lambda_{k-1}: \mathbb{R}^{k-2} \times \mathbb{R}^{\frac{(k-1)(k-2)}{2}} \rightarrow \mathbb{R}^{(k-1) \times(k-2)}
$$

such that

$$
A_{k-1}=\Lambda_{k-1}\left(\alpha_{k-2}, \lambda_{k-1}(\Omega)\right),
$$

and that for every $\lambda \in \mathbb{R}^{\frac{(k-1)(k-2)}{2}}$, the function $\alpha \mapsto \Lambda_{k-1}(\alpha, \lambda)$ is linear, and for every $\alpha \in \mathbb{R}^{k-2}$, the function $\lambda \mapsto \Lambda_{k-1}(\alpha, \lambda)$ is a polynomial of degree $k-2$. We want to define $\Lambda_{k}$.

The matrix $A_{k}$ can be written as a block matrix

$$
A_{k}=\left(\begin{array}{cc}
\beta_{k} & A_{k-1} \\
a_{1}^{(k-1)} & \gamma_{k}^{T}
\end{array}\right)
$$


where

$$
\beta_{k}=\left(\begin{array}{c}
a_{-2 k+3}^{(k-1)} \\
a_{-2 k+5}^{(k-1)} \\
\vdots \\
a_{-1}^{(k-1)}
\end{array}\right) \in \mathbb{R}^{k-1}, \quad \gamma_{k}=\left(\begin{array}{c}
a_{3}^{(k-2)} \\
\vdots \\
a_{2 k-5}^{(2)} \\
a_{2 k-3}^{(1)}
\end{array}\right) \in \mathbb{R}^{k-2}
$$

Thanks to formula (5.8), we can express all the entries of $\beta_{k}$ in terms of the entries of $A_{k-1}$ and of $\alpha_{k-1}$. We can therefore define a linear map $\widetilde{\beta}_{k}$ : $\mathbb{R}^{(k-1) \times(k-2)} \times \mathbb{R}^{k-1} \rightarrow \mathbb{R}^{k-1}$ such that

$$
\beta_{k}=\widetilde{\beta}_{k}\left(A_{k-1}, \alpha_{k-1}\right) \text {. }
$$

Then we can use (5.9) to express all the elements of $\gamma_{k}$ in terms of the entries of $A_{k-1}$ and of the elements of $\lambda_{k}(\Omega)$. In particular, we can define a bilinear $\operatorname{map} \widetilde{\gamma}_{k}: \mathbb{R}^{(k-1) \times(k-2)} \times \mathbb{R}^{\frac{k(k-1)}{2}} \rightarrow \mathbb{R}^{k-1}$ such that

$$
\gamma_{k}=\widetilde{\gamma}_{k}\left(A_{k-1}, \lambda_{k}(\Omega)\right)
$$

Finally, we use again formula (5.9) to express $a_{1}^{(k-1)}$ in terms of the elements of $\beta_{k}$ and of $\lambda_{k}(\Omega)$. Hence there is a bilinear function $\widetilde{a}_{k}: \mathbb{R}^{(k-1)} \times \mathbb{R}^{\frac{k(k-1)}{2}} \rightarrow \mathbb{R}$ such that

$$
a_{1}^{(k-1)}=\widetilde{a}_{k}\left(\beta_{k}, \lambda_{k}(\Omega)\right) .
$$

In conclusion, applying the inductive hypothesis:

$$
\begin{aligned}
A_{k}= & \left(\begin{array}{cc}
\widetilde{\beta}_{k}\left(A_{k-1}, \alpha_{k-1}\right) & A_{k-1} \\
\widetilde{a}_{k}\left(\widetilde{\beta}_{k}\left(A_{k-1}, \alpha_{k-1}\right), \lambda_{k}(\Omega)\right) & \widetilde{\gamma}_{k}\left(A_{k-1}, \lambda_{k}(\Omega)\right)^{T}
\end{array}\right) \\
& =\left(\begin{array}{cc}
\widetilde{\beta}_{k}\left(\Lambda_{k-1}\left(\alpha_{k-2}, \lambda_{k-1}(\Omega)\right), \alpha_{k-1}\right) & \Lambda_{k-1}\left(\alpha_{k-2}, \lambda_{k-1}(\Omega)\right) \\
\widetilde{a}_{k}\left(\widetilde{\beta}_{k}\left(\Lambda_{k-1}\left(\alpha_{k-2}, \lambda_{k-1}(\Omega)\right), \alpha_{k-1}\right), \lambda_{k}(\Omega)\right) & \widetilde{\gamma}_{k}\left(\Lambda_{k-1}\left(\alpha_{k-2}, \lambda_{k-1}(\Omega)\right), \lambda_{k}(\Omega)\right)^{T}
\end{array}\right) \\
& =: \Lambda_{k}\left(\alpha_{k-1}, \lambda_{k}(\Omega)\right)
\end{aligned}
$$

Notice that for every $\lambda \in \mathbb{R}^{\frac{k(k-1)}{2}}$, the function $\alpha \mapsto \Lambda_{k}(\alpha, \lambda)$ is linear, and for every $\alpha \in \mathbb{R}^{k-1}$ the function $\lambda \mapsto \Lambda_{k}(\alpha, \lambda)$ is a polynomial of degree $k-1$.

Eventually, we can apply what we proved to formula (5.7), concluding that

$$
\begin{aligned}
\frac{\mathrm{d}^{k} \mathcal{E}}{\mathrm{d} s^{k}}(0) & =E_{k}\left(A_{k}, \alpha_{k}\right)+F_{k}\left(\alpha_{k}, \alpha_{k}\right) \\
& =E_{k}\left(\Lambda_{k}\left(\alpha_{k-1}, \lambda_{k}(\Omega)\right), \alpha_{k}\right)+F_{k}\left(\alpha_{k}, \alpha_{k}\right) \\
& =: \Psi_{k}\left(\alpha_{k}, \lambda_{k}(\Omega)\right)
\end{aligned}
$$

The map $\Psi_{k}: \mathbb{R}^{k} \times \mathbb{R}^{\frac{k(k-1)}{2}} \rightarrow \mathbb{R}$ defined as above satisfies the requested properties.

Remark 5.4. The proof of Theorem 1.1 is constructive. In particular it allows us to obtain $\Lambda_{k}$ from $\Lambda_{k-1}$ using only elementary computations. In this way for every $k \geq 2$ we can find an explicit expression for $\frac{\mathrm{d}^{k} \mathcal{E}}{\mathrm{d} s^{k}}(0)$ in terms of 
$a_{1}, \ldots, a_{2 k-1}$ and of the coefficients in (5.5). We write here the list of the first three derivatives of the energy:

$$
\begin{aligned}
\frac{\mathrm{d} \mathcal{E}}{\mathrm{d} s}(0)= & -\frac{\pi}{4} a_{1}^{2} \\
\frac{\mathrm{d}^{2} \mathcal{E}}{\mathrm{d} s^{2}}(0)= & \frac{\pi}{4} a_{1}^{2} c_{1}^{(1)}(\Omega)-\frac{3}{4} \pi a_{1} a_{3}, \\
\frac{\mathrm{d}^{3} \mathcal{E}}{\mathrm{d} s^{3}}(0)= & \frac{9}{8} \pi a_{1} a_{3} c_{1}^{(1)}(\Omega)+\left(-\frac{3}{8}\left(c_{1}^{(1)}(\Omega)\right)^{2}+\frac{9}{16} c_{3}^{(1)}(\Omega)+\frac{3}{16} c_{1}^{(2)}(\Omega)\right) \pi a_{1}^{2} \\
& -\frac{15}{8} \pi a_{1} a_{5}-\frac{9}{8} \pi a_{3}^{2} .
\end{aligned}
$$

\section{Acknowledgements}

This material is based on work supported by the Italian Ministry of Education, University, and Research under the Project "Calculus of Variations" (PRIN 2010-11) and by the European Research Council under Grant No. 290888 "Quasistatic and Dynamic Evolution Problems in Plasticity and Fracture". The first and third authors are members of the Gruppo Nazionale per l'Analisi Matematica, la Probabilità e le loro Applicazioni (GNAMPA) of the Istituto Nazionale di Alta Matematica (INdAM).

\section{References}

[1] Amestoy, M., Leblond, J.-B.: Crack paths in plane situations-II: detailed form of the expansion of the stress intensity factors. Int. J. Solids Struct. 29, 465$501(1992)$

[2] Bourdin, B., Francfort, G.A., Marigo, J.-J.: The variational approach to fracture. J. Elast. 91, 5-148 (2008)

[3] Grisvard, P.: Singularities in Boundary Value Problems. In: Recherches en Mathématiques Appliquées [Research Notes in Applied Mathematics], vol. 22. Springer, Masson, Paris

[4] Irwin, G.R.: Analysis of stresses and strains near the end of a crack traversing a plate. Trans. ASME J. Appl. Mech. 24, 361-364 (1957)

[5] Leblond, J.-B.: Crack paths in plane situations-I. General form of the expansion of the stress intensity factors. Int. J. Solids Struct. 25, 1311-1325 (1989)

Gianni Dal Maso and Gianluca Orlando

SISSA

Via Bonomea 265

34136 Trieste

Italy

e-mail: dalmaso@sissa.it 
Gianluca Orlando

e-mail: gianluca.orlando@sissa.it

Rodica Toader

DIMI

Università di Udine

Via delle Scienze 206

33100 Udine

Italy

e-mail: toader@uniud.it

Received: 10 March 2014.

Accepted: 1 October 2014. 